\title{
Basic Properties and Qualitative Dynamics of a Vector-Borne Disease Model with Vector Stages and Vertical Transmission
}

\author{
Sansao A. Pedro \\ Departamento de Matemática e Informática, Universidade Eduardo Mondlane, 257, Maputo, Mozambique \\ Correspondence should be addressed to Sansao A. Pedro; sansaopedro@gmail.com
}

Received 7 July 2018; Revised 30 September 2018; Accepted 10 October 2018; Published 1 November 2018

Academic Editor: Zhidong Teng

Copyright (C) 2018 Sansao A. Pedro. This is an open access article distributed under the Creative Commons Attribution License, which permits unrestricted use, distribution, and reproduction in any medium, provided the original work is properly cited.

\begin{abstract}
This work systematically discusses basic properties and qualitative dynamics of vector-borne disease models, particularly those with vertical transmission in the vector population. Examples of disease include Dengue and Rift Valley fever which are endemic in Sub-Saharan Africa, and understanding of the dynamics underlying their transmission is central for providing critical informative indicators useful for guiding control strategies. Of particular interest is the applicability and derivation of relevant population and epidemic thresholds and their relationships with vertical infection. This study demonstrates how the failure of $R_{0}$ derived using the next-generation method compounds itself when varying vertical transmission efficiency, and it shows that the host type reproductive number gives the correct $R_{0}$. Further, novel relationships between the host type reproductive number, vertical infection, and ratio of female mosquitoes to host are established and discussed. Analytical results of the model with vector stages show that the quantities $Q_{0}, Q_{0}^{v}$, and $R_{0}^{c}$, which represent the vector colonization threshold, the average number of female mosquitoes produced by a single infected mosquito, and effective reproductive number, respectively, provide threshold conditions that determine the establishment of the vector population and invasion of the disease. Numerical simulations are also conducted to confirm and extend the analytical results. The findings imply that while vertical infection increases the size of an epidemic, it reduces its duration, and control efforts aimed at reducing the critical thresholds $Q_{0}, Q_{0}^{v}$, and $R_{0}^{c}$ to below unity are viable control strategies.
\end{abstract}

\section{Introduction}

Vector-borne diseases have been the scourge of man and animals since the beginning of time [1]. Today, vector-borne diseases account for over $17 \%$ of all infectious diseases causing more than 1 million deaths annually, and their distribution is mainly determined by a complex dynamic of environmental and social factors [2]. In spite of all these inherent complexities, mathematical models have been used to translate assumptions concerning biological, environmental, and social aspects into mathematical structures, linking biological processes of transmission and dynamics of infection at population level. Such dynamic models have impacted both our understanding of epidemic spread and public health planning (for more details see [3-5] and references therein).

In this study our particular interest is in investigating qualitative properties of epidemic models of mosquitoborne diseases in which the vector is of genera Aedes.
These mosquito species are known to transmit many vectorborne diseases of vast epidemiological importance including Dengue fever and Rift Valley fever (RVF), just to mention a few. These diseases are endemic in Sub-Saharan Africa with pronounced health and economic impacts on domestic animals and humans. In fact, losses due to RVF can reach millions of dollars during periods of disease outbreaks $[6,7]$. An interesting phenomenon underlying many vector-borne diseases is their ability to persist year round, fluctuating seasonally but only falling to zero during some months. Hence, an important question is, how does the virus maintain itself in nature? For RVF it has been hypothesized that RVF virus (RVFV) is maintained through transovarial transmission in Aedes mosquito eggs [8]. Aedes eggs need be dry for several days before they can mature. After maturing, they hatch during the next flooding event large enough to cover them with water [9-11]. The eggs have high desiccation resistance and can survive dry conditions in a dormant form 
for months to years [12-15]. Thus, the emergence of adult Aedes mosquitoes from infected eggs can reintroduce RVF in livestock at the beginning of the rainy season, before other mosquitoes species amplify it further [16]. For instance, in eastern and southern Africa there is more and more evidence of disease activities between outbreaks [7, 17-20] highlighting the role of vertical transmission for initial disease spread and endemicity.

In epidemiology disease spread and persistence are measured through quantities known as epidemic thresholds. Their derivation and characterization are one of the most important results of mathematical epidemic models. The basic reproductive number, $R_{0}$, is the most critical epidemic threshold given its applicability and suitability for deciding whether an outbreak will occur or fade out, making it essential for guiding disease control efforts. However, the derivation of this epidemic threshold in vector-borne disease models in particular suffers from a lack of uniqueness and it fails to give the correct average number of expected secondary infections produced by one infected individual [21]. This failure is more likely to compound itself when vertical transmission mode is included in the transmission model, since the resulting $R_{0}$ comes as the sum of the vertical and horizontal transmission components, if the nextgeneration method is used. Previous mathematical models have made a significant attempt in including vertical infection in modelling vector-borne diseases [22-28], but none of them discuss how the failure of $R_{0}$ compounds itself in the presence of vertical transmission. Therefore, the present work aims to discuss some relevant basic properties of vector-borne disease models when vertical infection is taken into account and their implications for disease control efforts. Further, our goal is to derive new epidemic thresholds useful for guiding control efforts in the settings of vector-borne disease models with vector stages that include vertical transmission mode.

We formulate two models, one simple but realistic and the other more complex with vector stages. The first is an extension of the one proposed by Ross [29] and popularized by Macdonald [30] and Anderson [31]. The model is used to discuss system properties such as the asymmetric relationship between the host-to-vector and vector-to-host reproductive numbers. In addition, we highlight how to derive epidemic thresholds useful for guiding disease control efforts and discuss their relationships with vertical transmission efficiency. It is shown that the model has two model equilibria, namely, the disease-free and the endemic, and Lyapunov function theory is used to establish their global qualitative dynamics. The second is an extension of the basic model, where the dynamics of both aquatic and adult mosquitoes are modelled explicitly. In this model we let the populations of aquatic and adults vary with time but be limited by their respective carrying capacity. The inclusion of the explicit vector submodel allows for derivation of critical thresholds such as the reproductive number for both the vector population and the disease system. Then, these thresholds are used to determine global qualitative dynamics of both the diseasefree and endemic equilibria.

The paper is set out as follows. In Section 2 we formulate and discuss the two model systems. In Section 3 we provide the epidemic threshold theorems regarding both the vector population and disease equilibria. We also discuss important model properties and how to derive and identify model epidemic thresholds useful for guiding disease control efforts. Furthermore, numerical simulations are carried out to investigate the influence of the key parameters on the spread of the disease (taking RVF as disease example), to support analytical analyses and conclusions and illustrate possible behavioural scenarios of the model with vector stages. Finally, in Section 4 we present a short discussion of the results and their biological implications.

\section{Materials and Methods}

For human and animal diseases, horizontal transmission typically occurs through direct or indirect physical contact with infectious hosts, or through disease vectors such as mosquitoes, ticks, or other biting insects. Among mosquito vectors vertical transmission is often through eggs. Of particular interest are female mosquitoes of genera Aedes which transmit the virus to their eggs. These eggs have some adaptive behaviour which allows them to stay dormant in nature for relatively long periods. Although vertical transmission also occurs among vectors involved in the transmission of Dengue disease, RVF is the disease for which the model is a good approximation. In particular, parameter values related to RVF are used to illustrate the dynamics of the disease numerically.

2.1. Host-Vector Basic Model with Vertical Transmission. Let $N_{h}=S_{h}+I_{h}+R_{h}$ and $N_{v}=S_{v}+I_{v}$ denote the total host and vector populations sizes, respectively. We assume that individuals at each compartment mix homogeneously and each mosquito bites each individual host at a constant rate $\alpha / N_{h}$, where $\alpha$ is the biting rate per unit time. Let $p_{h v}$ be a probability of successful infection transmission from an infected mosquito to a susceptible host and $p_{v h}$ be a probability of successful infection transmission from an infected host to a susceptible mosquito per bite. Thus, the forces of infection are as follows, $\lambda_{h v}=p_{h v}\left(\alpha / N_{h}\right) I_{v}$ and $\lambda_{v h}=$ $p_{h v}\left(\alpha / N_{h}\right) I_{h}$. Hosts are recruited into the population at per capita rate $\mu_{h}$ which is proportional to the total population and leave each compartment through death. Noninfected mosquitoes join the susceptible compartment at rate $\mu_{v}\left(N_{v}-\right.$ $q_{v} I_{v}$ ) while vertically infected mosquitoes join the infected class at rate $\mu_{v} q_{v} I_{v}$. Assuming constant population sizes, that is, births equal to deaths, $R_{h}$ can be obtained when both $S_{h}$ and $I_{h}$ are known. In the same way, $S_{v}$ can be obtained when $I_{v}$ is known. Thus, the expressions for both $R_{h}$ and $S_{v}$ can be omitted and the system can be written in terms of proportions:

$$
\begin{aligned}
& \frac{d S_{h}}{d t}=\mu_{h}\left(1-S_{h}\right)-p_{h v} \alpha m I_{v} S_{h} \\
& \frac{d I_{h}}{d t}=p_{h v} \alpha m I_{v} S_{h}-\left(\gamma_{h}+\mu_{h}\right) I_{h} \\
& \frac{d I_{v}}{d t}=p_{v h} \alpha I_{h}\left(1-I_{v}\right)-\mu_{v}\left(1-q_{v}\right) I_{v}
\end{aligned}
$$


where $q_{v} \in(0,1)$ is the probability of vertical infection, $\gamma_{h}$ the rate at which infected hosts recover from infection, and $m$ denotes the ratio of female mosquitoes to hosts.

2.2. Model with Vector Stages and Vertical Transmission. Here we extend the basic model to include vector stages. Partial results of the resulting model without vertical infection have been obtained in [32]. Our aim is to extend their model and analysis by investigating global dynamics of all model equilibria and examine the extent to which vertical infection alters the dynamics of the system. The mosquito population is divided into aquatic (eggs, larvae, and pupae) and terrestrial (adults) subpopulations with $K_{1}$ and $K_{2}$ being their carrying capacity, respectively. The parameter $K_{1}$ represents the larval maximal capacity limited by the availability of breeding sites while $K_{2}$ is the maximal capacity of adult mosquitoes limited by factors conditioning their survival such as high altitudes and high temperatures. Further, the aquatic subpopulation is divided into epidemiological classes, susceptible $\left(L_{s}\right)$, and infected $\left(L_{i}\right)$ while adults are divided also into susceptible $\left(S_{v}\right)$ and infected $\left(I_{v}\right)$. The per capita oviposition rate is $r\left(1-L / K_{1}\right)$, where $r$ is the intrinsic oviposition rate and $L=L_{s}+L_{i}$. Aquatic mosquitoes emerge as adults at a per capita rate $\theta$ where the proportion $1-q_{v}$ emerge noninfected while the remainder are infected. Disease transmission dynamics between vector and host populations remain the same as in the basic model. As a result the following nondimensional system of ordinary differential equations represents the model that governs the temporal evolution of the disease:

$$
\begin{aligned}
& \frac{d L_{s}}{d t}=\delta r(1-L)\left(N_{v}-q_{v} I_{v}\right)-\left(\mu_{L}+\theta\right) L_{s}, \\
& \frac{d L_{i}}{d t}=\delta r(1-L) q_{v} I_{v}-\left(\mu_{L}+\theta\right) L_{i}, \\
& \frac{d S_{v}}{d t}=\frac{\theta}{\delta} L_{s}\left(1-N_{v}\right)-\lambda_{v h} I_{h} S_{v}-\mu_{v} S_{v}, \\
& \frac{d I_{v}}{d t}=\frac{\theta}{\delta} L_{i}\left(1-N_{v}\right)+\lambda_{v h} I_{h} S_{v}-\mu_{v} I_{v}, \\
& \frac{d S_{h}}{d t}=\mu_{h}\left(1-S_{h}\right)-\lambda_{h v} I_{v} S_{h}, \\
& \frac{d I_{h}}{d t}=\lambda_{h v} I_{v} S_{h}-\left(\gamma_{h}+\mu_{h}\right) I_{h},
\end{aligned}
$$

where $L=L_{s}+L_{i}, \delta=K_{2} / K_{1}, \lambda_{v h}=p_{v h} \alpha, \lambda_{h v}=p_{h v} \alpha m$, $m=K_{2} / N_{h}, S_{v}=S_{v} / K_{2}$, and $I_{v}=I_{v} / K_{2}$.

Let $\left\{\left\{L_{s}(0), L_{i}(0), S_{v}(0), I_{v}(0), S_{h}(0), I_{h}(0) \geqslant 0\right\} \in \mathbb{R}_{+}^{6}\right\}$ be the initial conditions of system (2). It is easy to check that the feasible region for (2) is the positive orthant of $\mathbb{R}^{6}$ and that the closed set

$$
\begin{aligned}
\Phi & =\left\{\left(L_{s}, L_{i}, S_{v}, I_{v}, S_{h}, I_{h}\right) \in \mathbb{R}_{+}^{6}: L_{s}+L_{i} \leqslant 1, S_{v}+I_{v}\right. \\
& \left.\leqslant 1, S_{h}+I_{h} \leqslant 1\right\}
\end{aligned}
$$

is positively invariant for system (2).

\subsubsection{Positivity of Solutions}

Lemma 1 (see [33]). Let us denote $u=\left(u_{i}\right)_{i=\overline{1, n}}$ and consider the function $f: \mathbb{R}_{+} \times \mathbb{R}^{n} \longrightarrow \mathbb{R}^{n}$ continuous with respect to $t, u$ and Lipschitz with respect to $u$. If $f(t, u) \geq 0$ for $(t, u) \in$ $\mathbb{R}_{+} \times \mathbb{R}^{n}$, with $u_{i}=0$, then, for every $u_{0} \in \mathbb{R}_{+}^{n}$, there exists $T>0$ such that the solution to

$$
\begin{aligned}
\frac{d u}{d t} & =f(t, u), \\
u\left(t_{0}\right) & =u_{0}
\end{aligned}
$$

exists and is unique and positive with value in $\mathbb{R}_{+}^{n}$ and defined on some interval $[0, T)$. If $T<\infty$, then

$$
\limsup _{t \rightarrow T} \sum_{i=1}^{n} u_{i}=+\infty .
$$

Theorem 2. The solution set $\left\{L_{s}(t), L_{i}(t), S_{v}(t), I_{v}(t), S_{h}(t)\right.$, $\left.I_{h}(t)\right\}$ of the model (2) exists and is unique and positive for $t>0$.

Proof. Let $X=\left(L_{s}(t), L_{i}(t), S_{v}(t), I_{v}(t), S_{h}(t), I_{h}(t)\right)$ and denote the function $f=\left(f_{i}\right)_{i=\overline{1,6}}$ such that

$$
\begin{aligned}
f_{1} & \left(L_{s}(t), L_{i}(t), S_{v}(t), I_{v}(t), S_{h}(t), I_{h}(t)\right) \\
= & \delta r(1-L)\left(N_{v}-q_{v} I_{v}\right)-\left(\mu_{L}+\theta\right) L_{s}, \\
f_{2} & \left(L_{s}(t), L_{i}(t), S_{v}(t), I_{v}(t), S_{h}(t), I_{h}(t)\right) \\
& =\delta r(1-L) q_{v} I_{v}-\left(\mu_{L}+\theta\right) L_{i}, \\
f_{3} & \left(L_{s}(t), L_{i}(t), S_{v}(t), I_{v}(t), S_{h}(t), I_{h}(t)\right) \\
& =\frac{\theta}{\delta} L_{s}\left(1-N_{v}\right)-\lambda_{v h} I_{h} S_{v}-\mu_{v} S_{v}, \\
f_{4} & \left(L_{s}(t), L_{i}(t), S_{v}(t), I_{v}(t), S_{h}(t), I_{h}(t)\right) \\
& =\frac{\theta}{\delta} L_{i}\left(1-N_{v}\right)+\lambda_{v h} I_{h} S_{v}-\mu_{v} I_{v}, \\
f_{5} & \left(L_{s}(t), L_{i}(t), S_{v}(t), I_{v}(t), S_{h}(t), I_{h}(t)\right) \\
& =\mu_{h}\left(1-S_{h}\right)-\lambda_{h v} I_{v} S_{h}, \\
f_{6} & \left(L_{s}(t), L_{i}(t), S_{v}(t), I_{v}(t), S_{h}(t), I_{h}(t)\right) \\
& =\lambda_{h v} I_{v} S_{h}-\left(\gamma_{h}+\mu_{h}\right) I_{h} .
\end{aligned}
$$

Since the function $f$ is continuous and Lipschitz continuous with respect to $X$, according to Picard's theorem, there exists $T_{0}>0$ such that the solution to (2) exists locally at least on an interval of this form $\left[0, T_{0}\right]$. Further, considering the initial condition $X_{1}=X\left(T_{0}\right)$ at $t_{0}=T_{0}$ and using Picard's theorem, it follows that there exists $T_{0} \leq T_{1} \in R_{+}$such that the solution to (2) exists and is unique on $\left[T_{0}, T_{1}\right]$. Since $f$ is continuous and differentiable, the solution of (2) with a given initial condition is unique. Therefore, the solutions of (2) obtained on $\left[0, T_{0}\right]$ and on $\left[T_{0}, T_{1}\right]$ form the unique solution of $(2)$ on $\square_{T_{1}}=\left[0, T_{1}\right]$ with the initial condition $X_{0}$ at 
$t_{0}=0$. Repeating this process again and again, we end up with the maximal forward interval of existence for the solutions of (2), say $\rrbracket_{\widehat{T}}=[0, \widehat{T})$ with $\widehat{T}>0$. Furthermore, for $X \in \mathbb{R}_{+}^{6}$,

$$
f_{i}\left(L_{s}(t), L_{i}(t), S_{v}(t), I_{v}(t), S_{h}(t), 0\right) \geq 0 .
$$

Therefore, the solutions of (2) on $\rrbracket_{\widehat{T}}$ are positive, according to Lemma 1.

Finally, according to Theorem 2, the solutions to (2) are bounded on $[0, \widehat{T})$. In other words, they do not blow up on any finite interval of $\mathbb{R}_{+}$. It follows that, according to Lemma 1 , the solution of (2) exists for all time. Hence for any initial condition in $\mathbb{R}_{+}^{6}$, system (2) possesses a unique and positive solution in $\mathbb{R}_{+}^{6}$.

\section{Results}

\subsection{Analysis of the Basic Host-Vector Model}

3.1.1. Model Equilibria and Stability Analysis. The basic hostvector model with vertical transmission exhibits two equilibria, namely, the disease-free $E_{1}^{0}$ and the endemic $E_{1}^{*}$, respectively. At the disease-free equilibrium,

$$
E_{1}^{0}=\left(S_{h}^{0}, I_{h}^{0}, I_{v}^{0}\right)=(1,0,0),
$$

both vector and host populations persist but with no disease. The prevalence of the disease is denoted by

$$
\begin{aligned}
E_{1}^{*} & =\left(S_{h}^{*}, I_{h}^{*}, I_{v}^{*}\right) \\
& =\left(\frac{a+\mu_{h} R_{0}^{e}}{\left(a+\mu_{h}\right) R_{0}^{h}}, \frac{\mu_{h}\left(R_{0}^{e}-1\right)}{c R_{0}^{e}+b \mu_{h} / V}, \frac{\mu_{h}\left(R_{0}^{e}-1\right)}{a+\mu_{h} R_{0}^{e}}\right),
\end{aligned}
$$

where $a=p_{h v} \alpha m, b=p_{v h} \alpha, c=\gamma_{h}+\mu_{h}, V=\mu_{v}\left(1-q_{v}\right)$, and

$$
R_{0}^{e}=\frac{a b}{V c}=\frac{p_{h v} p_{v h} \alpha^{2} m}{\mu_{v}\left(1-q_{v}\right)\left(\gamma_{h}+\mu_{h}\right)} .
$$

The Jacobian matrix of system (1) at $E_{1}^{0}$ is given by

$$
J_{1}^{\prime}=\left(\begin{array}{ccc}
-\mu_{h} & 0 & -a \\
0 & -c & a \\
0 & b & -V
\end{array}\right)
$$

such that the characteristic polynomial of matrix (11) is then given as

$$
P_{1}(\lambda)=\lambda^{3}+a_{2} \lambda^{2}+a_{1} \lambda+a_{0},
$$

with $a_{2}=V+c+\mu_{h}, a_{1}=V c\left(1-R_{0}^{e}\right)+(V+c) \mu_{h}$, and $a_{0}=\mu_{h} V c\left(1-R_{0}^{e}\right)$. The coefficient $a_{2}>0$ and both $a_{1}, a_{0}$ are nonnegative if and only if $R_{0}^{e}<1$. Hence, all Routh stability criteria are satisfied; that is, the three eigenvalues of matrix (11) are negative or have negative real parts. Furthermore, for $R_{0}^{e}=1$, (9) becomes the disease-free equilibrium. Therefore, the following result holds.

Theorem 3. The disease-free equilibrium $E_{1}^{0}=(1,0,0)$ exists and it is globally asymptotically stable if $R_{0}^{e} \leqslant 1$.
Alternatively, the global stability of the disease-free equilibrium $E_{1}^{0}$ can be established using the following Lyapunov function:

$$
V\left(S_{h}, I_{h}, I_{v}\right)=u_{1}\left(S_{h}-S_{h}^{0}-S_{h}^{0} \ln \frac{S_{h}}{S_{h}^{0}}\right)+u_{2} I_{h}+u_{3} I_{v}
$$

where $u_{1}, u_{2}, u_{3}$ are some positive constants. Calculating the derivative of $V$ along the solutions of system (1), we obtain

$$
\begin{aligned}
V^{\prime} & =u_{1} \frac{S_{h}-S_{h}^{0}}{S_{h}}\left[\mu_{h}\left(S_{h}^{0}-S_{h}\right)-a S_{h} I_{v}\right] \\
& +u_{2}\left[a S_{h} I_{v}-\left(\gamma_{h}+\mu_{h}\right) I_{h}\right] \\
& +u_{3}\left[b I_{h}\left(1-I_{v}\right)-\mu_{v}\left(1-q_{v}\right) I_{v}\right], \\
= & -u_{1} \mu_{h} \frac{\left(S_{h}^{0}-S_{h}\right)^{2}}{S_{h}} \\
& -u_{3} \mu_{v}\left(1-q_{v}\right)\left[1-\frac{u_{1} a}{u_{3} \mu_{v}\left(1-q_{v}\right)}\right] I_{v} \\
& -u_{2}\left(\gamma_{h}+\mu_{h}\right)\left[1-\frac{u_{3} b}{u_{2}\left(\gamma_{h}+\mu_{h}\right)}\right] I_{h} \\
- & u_{3} b I_{h} I_{v}+\left(u_{2}-u_{1}\right) a S_{h} I_{v} .
\end{aligned}
$$

Choosing $u_{1}=u_{2}=b /\left(\gamma_{h}+\mu_{h}\right)$ and $u_{3}=1$, (16) becomes

$$
\begin{aligned}
V^{\prime}= & -u_{1} \mu_{h} \frac{\left(S_{h}^{0}-S_{h}\right)^{2}}{S_{h}}-\mu_{v}\left(1-q_{v}\right)\left[1-R_{0}^{e}\right] I_{v} \\
& -b I_{h} I_{v} .
\end{aligned}
$$

Thus, $V^{\prime}$ is negative for $R_{0}^{e} \leqslant 1$. Note also that $V^{\prime}=0$ if and only if $S_{h}=S_{h}^{0}$ and $I_{h}=I_{v}=0$. Therefore, the largest invariant set for (1) is the singleton $\left\{E_{1}^{0}\right\}$. Hence, by LaSalle's invariance principle [34], $E_{1}^{0}$ is globally asymptotically stable when $R_{0}^{e} \leqslant$ 1 and Theorem 3 is valid.

Remark 4. Clearly, the endemic equilibrium $E_{1}^{*}$ exists and is unique for $R_{0}^{e}>1$. This excludes the possibility of occurrence of backward bifurcation. This result is of great epidemiological significance in guiding efforts for disease control as it indicates that $R_{0}^{e}=1$ is the critical epidemic threshold.

To establish the local stability of the endemic equilibrium we evaluate the Jacobian of the system at $E_{1}^{*}$, which gives

$$
J_{1}^{*}=\left(\begin{array}{ccc}
-\left(\mu_{h}+a I_{v}^{*}\right) & 0 & -a S_{h}^{*} \\
a I_{v}^{*} & -c & a S_{h}^{*} \\
0 & b\left(1-I_{v}^{*}\right) & -b I_{h}^{*}-V
\end{array}\right) \text {. }
$$

The characteristic polynomial of matrix (18) is then given by

$$
P_{2}(\lambda)=\lambda^{3}+b_{2} \lambda^{2}+b_{1} \lambda+b_{0},
$$


where

$$
\begin{aligned}
b_{2}= & V^{2} c R_{0}^{e}\left(\mu_{h}+a\right)+V c^{2} R_{0}^{e}\left(R_{0}^{e} \mu_{h}^{2}+a\right) \\
& +V a R_{0}^{e} \mu_{h}\left(v R_{0}^{e}+b\right)+V b\left(R_{0}^{e}\right)^{2} \mu_{h} \\
& +b \mu_{h}^{2} R_{0}^{e}\left(a+c+\mu_{h}\right)+a b c \mu_{h} \\
b_{1}= & V^{2} c\left(R_{0}^{e}\right)^{3} \mu_{h}\left(a+c+\mu_{h}\right)+V b\left(R_{0}^{e}\right)^{3} \mu_{h}^{2}\left(a+\mu_{h}\right) \\
& +V c^{2}\left(R_{0}^{e}\right)^{3} \mu_{h}\left(a+\mu_{h}\right) \\
& +V b c\left(R_{0}^{e}\right)^{2} \mu_{h}^{2}\left(R_{0}^{e}-1\right) \\
& +a b c R_{0}^{e} \mu_{h}^{2}\left(R_{0}^{e}-\frac{V}{\mu_{h}}\right)+b c\left(R_{0}^{e}\right)^{2} \mu_{h}^{3}, \\
b_{0}= & V^{2} c^{2}\left(R_{0}^{e}\right)^{3} \mu_{h}+V b c\left(R_{0}^{e}\right)^{2} \mu_{h}^{2}\left(R_{0}^{e}-1\right) \\
& +V^{2} a c^{2}\left(R_{0}^{e}\right)^{2}\left(R_{0}^{e}-1\right) \\
& +V a b c R_{0}^{e} \mu_{h}\left[R_{0}^{e}\left(R_{0}^{e}-1\right)-1\right] .
\end{aligned}
$$

The coefficient $b_{2}>0$ and both $b_{1}, b_{0}$ are nonnegative if and only if $R_{0}^{e}>1$. Hence, all Routh criteria are satisfied; that is, the three eigenvalues of matrix (11) are negative or have negative real parts. Therefore, the following results holds.

Theorem 5. The endemic equilibrium $E_{1}^{*}=\left(S_{h}^{*}, I_{h}^{*}, I_{v}^{*}\right)$ exists and it is locally asymptotically stable if $R_{0}^{e}>1$.

A global stability result for the endemic equilibrium $E_{1}^{*}$ of system (1) is given below.

Theorem 6. If $R_{0}^{e}>1$, the endemic equilibrium $E_{1}^{*}=\left(S_{h}^{*}\right.$, $\left.I_{h}^{*}, I_{v}^{*}\right)$ is globally asymptotically stable.

Proof. Let $D_{1}=v_{1}\left(S_{h}-S_{h}^{*}-S_{h}^{*} \ln \left(S_{h} / S_{h}^{*}\right)\right), D_{2}=v_{2}\left(I_{h}-\right.$ $\left.I_{h}^{*}-I_{h}^{*} \ln \left(I_{h} / I_{h}^{*}\right)\right)$, and $D_{3}=v_{3}\left(I_{v}-I_{v}^{*}-I_{v}^{*} \ln \left(I_{v} / I_{v}^{*}\right)\right)$ be the components of the Lyapunov function

$$
U\left(S_{h}, I_{h}, I_{v}\right)=D_{1}+D_{2}+D_{3},
$$

where $v_{1}, v_{2}, v_{3}$ are some positive parameters to be chosen later. Differentiating $U$ along the solutions of system (1), we obtain

$$
\begin{aligned}
D_{1}^{\prime} & =v_{1} \frac{S_{h}-S_{h}^{*}}{S_{h}}\left[\mu_{h}-\mu_{h} S_{h}-a S_{h} I_{v}\right] \\
& =-v_{1} \mu_{h} \frac{\left(S_{h}-S_{h}^{*}\right)^{2}}{S_{h}}+v_{1} \frac{S_{h}-S_{h}^{*}}{S_{h}}\left(a S_{h}^{*} I_{v}^{*}-a S_{h} I_{v}\right) \\
& =-v_{1} \mu_{h} \frac{\left(S_{h}-S_{h}^{*}\right)^{2}}{S_{h}}+I_{v}^{*}-\frac{S_{h}}{S_{h}^{*}} I_{v}-\frac{S_{h}^{*}}{S_{h}} I_{v}^{*}-I_{v},
\end{aligned}
$$

for $v_{1}=1 / a S_{h}^{*}$ and $\mu_{h}=\mu_{h} S_{h}^{*}-a S_{h}^{*} I_{v}^{*}$ at equilibrium.

$$
D_{2}^{\prime}=v_{2} \frac{I_{h}-I_{h}^{*}}{I_{h}}\left[a S_{h} I_{v}-\left(\gamma_{h}+\mu_{h}\right) I_{h}\right]
$$

$$
\begin{aligned}
& =v_{2} \frac{I_{h}-I_{h}^{*}}{I_{h}}\left[a S_{h} I_{v}-\frac{a S_{h}^{*} I_{v}^{*}}{I_{h}^{*}} I_{h}\right] \\
& =\frac{S_{h}}{S_{h}^{*}} I_{v}-\frac{I_{h}}{I_{h}^{*}} I_{v}^{*}-\frac{I_{h}^{*} S_{h}}{I_{h} S_{h}^{*}} I_{v}+I_{v}^{*},
\end{aligned}
$$

for $v_{2}=v_{1}$ and $\gamma_{h}+\mu_{h}=a S_{h}^{*} I_{v}^{*} / I_{h}^{*}$ at equilibrium.

$$
\begin{aligned}
D_{3}^{\prime} & =v_{3} \frac{I_{v}-I_{v}^{*}}{I_{v}}\left[b I_{h}\left(1-I_{v}\right)-\mu_{v}\left(1-q_{v}\right) I_{v}\right] \\
& =b v_{3} \frac{I_{v}-I_{v}^{*}}{I_{v}}\left[b I_{h}\left(1-I_{v}\right)-\frac{I_{h}^{*}\left(1-I_{v}^{*}\right)}{I_{v}^{*}} I_{v}\right] \\
& =b v_{3} \frac{I_{v}-I_{v}^{*}}{I_{v}}\left[I_{h}-I_{h} I_{v}-I_{v} I_{h}^{*}\left(\frac{1-I_{v}^{*}}{I_{v}^{*}}\right)\right], \\
& \leqslant \frac{I_{v}-I_{v}^{*}}{I_{v}}\left[I_{h}-I_{h} I_{v}-I_{v} I_{h}^{*}\right],
\end{aligned}
$$

for $v_{3}=1 / b$ and $\mu_{v}\left(1-q_{v}\right)=I_{h}^{*}\left(1-I_{v}^{*}\right) / I_{v}^{*}$ at equilibrium. Now all together,

$$
\begin{aligned}
U^{\prime} \leqslant & -v_{1} \mu_{h} \frac{\left(S_{h}-S_{h}^{*}\right)^{2}}{S_{h}}-I_{v}^{*}\left(\frac{I_{v}}{I_{v}^{*}}+\frac{I_{h}}{I_{h}^{*}}-2\right)-\frac{S_{h}^{*}}{S_{h}} I_{v}^{*} \\
& -\frac{I_{h}^{*} S_{h}}{I_{h} S_{h}^{*}} I_{v}-I_{v}^{*}\left(\frac{I_{v}}{I_{v}^{*}}+\frac{I_{h}}{I_{h}^{*}}-2\right)-2 I_{v}^{*}+2 I_{v}+I_{h} \\
& -I_{h} I_{v}-I_{v} I_{h}^{*}-\frac{I_{v}^{*}}{I_{v}} I_{h}+I_{v}^{*}+I_{v}^{*} I_{h}^{*}, \\
\leqslant & -v_{1} \mu_{h} \frac{\left(S_{h}-S_{h}^{*}\right)^{2}}{S_{h}}-2 I_{v}^{*}\left(\frac{I_{v}}{I_{v}^{*}}+\frac{I_{h}}{I_{h}^{*}}-2\right) \\
& +2 I_{v}\left(1-\frac{I_{v}^{*}}{I_{v}}\right)+I_{h}\left(1-\frac{I_{v}^{*}}{I_{v}}\right) \\
& +I_{v}^{*} I_{h}^{*}\left(1-\frac{I_{v}}{I_{v}^{*}}\right) \\
& +I_{v}^{*}\left[1-\frac{S_{h}^{*}}{S_{h}}\left(1+\frac{I_{h}^{*} S_{h}^{2} I_{v}}{I_{h}\left(S_{h}^{*}\right)^{2} I_{v}^{*}}\right)\right] .
\end{aligned}
$$

Using the inequality $1-x+\ln x \leqslant 0$ for $x>0$ with equality holding if and only if $x=1$ and the fact that the arithmetic mean is greater than or equal to the geometric mean, we obtain $U^{\prime}\left(S_{h}, I_{h}, I_{v}\right) \leqslant 0$ for all $S_{h}, I_{h}, I_{v}>0$. Furthermore, we obtain that $U^{\prime}\left(S_{h}, I_{h}, I_{v}\right)=0$ holds only when $S_{h}=S_{h}^{*}, I_{h}=I_{h}^{*}, I_{v}=I_{v}^{*}$ and that $E_{1}^{*}$ is the only equilibrium state of these systems on this plane (line). Therefore, by LaSalle's invariance principle [34], the positive equilibrium $E_{1}^{*}$ is globally asymptotically stable.

3.1.2. Epidemic Thresholds, Vertical Infection, and Basic Properties. One of the most important critical thresholds in epidemic models is the basic reproductive number, $R_{0}$, which is 
usually found using the next-generation method, as the dominant eigenvalue of the next-generation matrix $[35,36]$. Following the method in [36] we write system (1) consisting only of infectious compartments as the difference between new infection and transfer rates and the resulting Jacobian matrices evaluated at the disease-free equilibrium $E^{0}=(1,0,0)$. For system (1) we have two infected classes, namely, $I_{v}$ and $I_{h}$. It follows that the transmission and the transfer matrices $F$ and $F$, respectively, are defined as

$$
\begin{aligned}
& F=\left(\begin{array}{cc}
q_{v} \mu_{v} & p_{v h} \alpha \\
p_{h v} \alpha m & 0
\end{array}\right), \\
& V=\left(\begin{array}{cc}
\mu_{v} & 0 \\
0 & \gamma_{h}+\mu_{h}
\end{array}\right) .
\end{aligned}
$$

Unlike in host-vector models without vertical transmission, the diagonal elements of the transmission matrix $F$ are nonzero. This stems from the fact that in the presence of vertical transmission there is vector to vector transmission, which completely changes the nature of the basic reproductive number. Thus, the next-generation matrix, $K$, is then given by

$$
K=F V^{-1}=\left(\begin{array}{cc}
q_{v} & \frac{p_{v h} \alpha}{\gamma_{h}+\mu_{h}} \\
\frac{p_{h v} \alpha m}{\mu_{v}} & 0
\end{array}\right)
$$

and the resulting dominant eigenvalue of the spectral radius $F V^{-1}$, which is the basic reproductive number, $R_{0}$, is given by

$$
\begin{array}{r}
R_{0}=\frac{1}{2} q_{v}+\frac{1}{2} \sqrt{q_{v}^{2}+4 R_{0}^{H}}, \\
\qquad \text { with } R_{0}^{H}=\frac{p_{v h} p_{h v} \alpha^{2} m}{\mu_{v}\left(\gamma_{h}+\mu_{h}\right)} .
\end{array}
$$

When there is no vertical transmission, $q_{v}=0$, as in the case of malaria, $R_{0}=R_{0}^{H}$ is simply the geometric mean of the product of the number of new infections in hosts from one infected vector and the number of new infections in vectors from one infected host, in the limiting case that both populations are fully susceptible. The interpretation and epidemiological significance of $R_{0}^{H}$ are well established. It is easy to see that the transmission of infection is increased with efficiency of vector biting $\alpha$ and probabilities of successful infection transmission, but it is hindered by high mosquito death rates and faster host recovery. The biting rate $\alpha$ appears as $\alpha^{2}$ because it enters twice in the transmission cycle [31]. An important parameter is the ratio of female mosquitoes to hosts $m=N_{v} / N_{h}$, which is central for disease spread according to model settings. $R_{0}$ increases with the number (or density) of mosquitoes but decreases with the number (or density) of host population. This results from the asymmetry in the dependence of the vector's biting rate on the sizes of the host and vector populations such that when there are many more hosts compared to mosquitoes, sustained transmission may be impossible. Therefore, in the absence of vertical transmission, for the infection to successfully spread and invade, the ratio of mosquitoes to hosts needs to be sufficiently large so that double bites are common [37]:

$$
\frac{N_{v}}{N_{h}}>\frac{\mu_{v}\left(\gamma_{h}+\mu_{h}\right)}{p_{v h} p_{h v} \alpha^{2}},
$$

where the critical ratio is given by $m_{c}=\mu_{v}\left(\gamma_{h}+\mu_{h}\right) / p_{v h} p_{h v} \alpha^{2}$. Note that each mosquito could infect less than one host on average, and yet $R_{0}$ could still be more than unity. To elucidate this fact we write $R_{0}^{H}$ as a product of each host type single-step reproductive number; that is,

$$
R_{0}^{H}=R_{h v} \times R_{v h}=\frac{p_{h v} \alpha m}{\mu_{v}} \times \frac{p_{v h} \alpha}{\gamma_{h}+\mu_{h}},
$$

where $R_{v h}$ represents the number of new infections in mosquitoes from a single infected host while $R_{h v}$ represents the number of new infections in hosts from a single infected mosquito. Clearly, $R_{0}^{H}$ can be greater than unity even when one of these reproduction numbers is less than unity, and it can also be less than unity even if one of its components is greater than unity. In Figure 1(a) we depict contours plots corresponding to the overall $R_{0}$ in (31) along the plane $\left(R_{h v}, R_{v h}\right)$ in the presence of vertical transmission. Its effects in this asymmetric relationship between $R_{0}$ and its components is not very pronounced and the ratio of mosquitoes to hosts remains one of the leading factors when there is a large disparity between the sizes of the host and vector populations.

Clearly, the geometric mean is less than the average expected number of new infections per generation. This is the case where the next-generation method fails to produce the correct $R_{0}$ if transmission between hosts is intermediated by another host (for more discussion about the failure of this method see [21]). Instead, it gives the weighted average lying between the number of new infections each individual produces in the next infection event. Note that if the number of hosts is increased, this deficit is compounded. This can have serious implications for guiding disease control efforts as it fails to provide the actual severity of the infection. Therefore, in this study for application purpose we propose the use of another epidemic threshold. This is $R_{0}$ from (10) derived in Section 3.1.1, which is hereby referred to as the 'effective' reproductive number:

$$
R_{0}^{e}=\frac{1}{1-q_{v}} \frac{p_{v h} p_{h v} \alpha^{2} m}{\mu_{v}\left(\gamma_{h}+\mu_{h}\right)},
$$

as (1) it satisfies the property that the endemic equilibrium $E_{1}^{*}$ only persists if $R_{0}^{e}$ is greater than unity and (2) the endemic equilibrium exists without occurrence of backward bifurcation, meaning that $R_{0}^{e}=1$ is the correct critical value. At this point, it is of particular interest to establish the critical ratio of mosquitoes to hosts in the settings of vertical transmission. From (31) we obtain

$$
\frac{N_{v}}{N_{h}}>\left(1-q_{v}\right) \frac{\mu_{v}\left(\gamma_{h}+\mu_{h}\right)}{p_{v h} p_{h v} \alpha^{2}},
$$

that is, a new critical ratio $m_{c}^{*}=\left(1-q_{v}\right) m_{c}<m_{c}$ for $0<q_{v}<1$. This result indicates that, in the presence of 


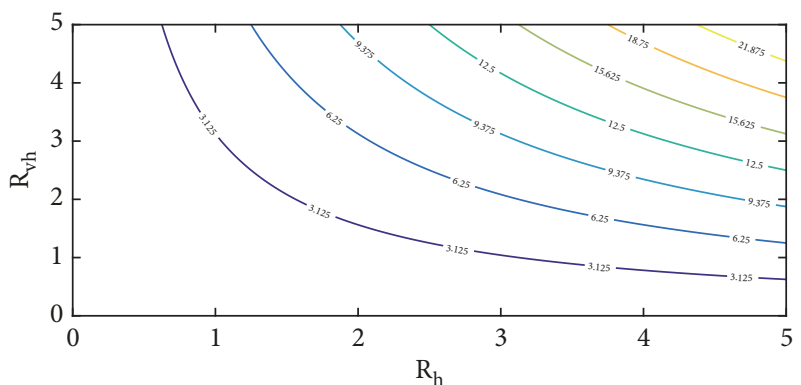

(a)

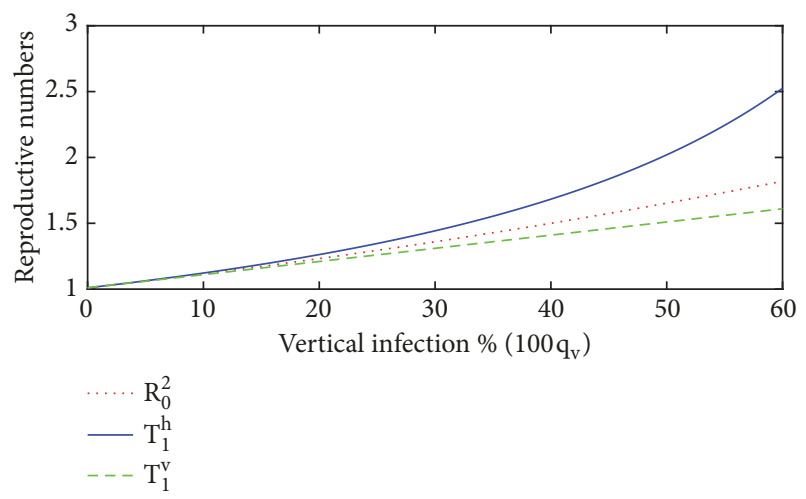

(c)

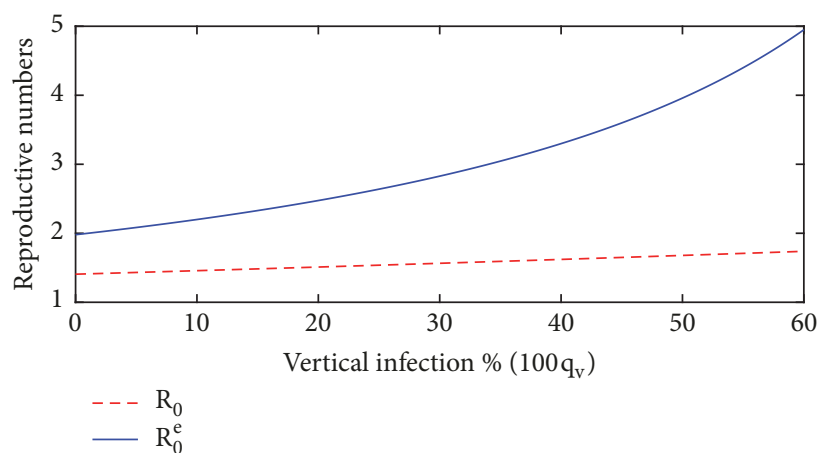

(b)

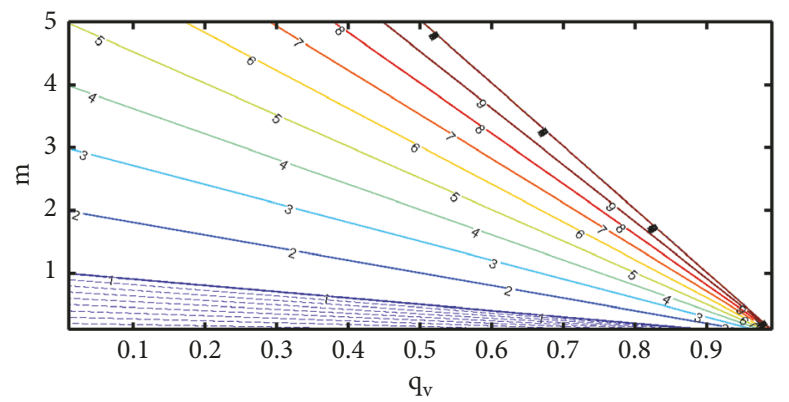

(d)

FIGURE 1: Relationships among epidemic thresholds with respect to vertical transmission and ratio of mosquitoes to hosts. (a) The relationship between number of new infections in hosts and number of new infections in vectors. (b) Comparison between basic reproductive numbers $R_{0}$ and $R_{0}^{e}$ derived from two different methods with respect to vertical infection. (c) Comparison of behaviours among the complete transmission cycle $R_{0}^{2}$ and host type and vector type reproductive numbers with respect to vertical transmission efficiency away from the common epidemic criterion. (d) How the ratio of mosquitoes to hosts $m$ and vertical infection $q_{v}$ contribute to patterns of $R_{0}^{e}$. Dashed lines indicated contours lines for $R_{0}^{e}<1$ while continuous lines are for $R_{0}^{e} \geqslant 1$.

vertical transmission, the critical ratio of female mosquitoes to hosts reduces at a rate proportional to vertical transmission efficiency. This indicates an earlier occurrence of the point where an epidemic is just possible as above this level the equilibrium prevalence is expected to rapidly increase to its asymptotic value.

Others are epidemic thresholds that provide a direct measure of the control effort required for disease eradication. These measures are known as "type" reproductive numbers and are determined based on the next-generation matrix $[38,39]$ such that for our case we have host and vector type reproductive numbers $T_{1}^{h}$ and $T_{1}^{v}$, respectively. Note that in the absence of vertical transmission the host and vector type reproductive numbers are both equal to the square of the basic reproductive number, $R_{0}$ in (41), which turns out to be $R_{0}^{e}$ in (31) for $q_{v}=0$. In the presence of vertical infection, $R_{0}^{2}$ gives the expected number of secondary infections after one average, complete (host-vector-host or vector-host-vector) transmission cycle but does not correspond to a specific population type [23]. For the case of host population, one infected host leads to some secondary host infections in the next host-vector-host transmission cycle. This results exactly from the horizontal transmission mechanism and it is given by $R_{0}^{H}$. However, further secondary host infections may also occur after any number of vector-vector transmission cycles as a result of transovarial transmission. From the expression of $R_{0}$ in (31) it can be seen that new infections resulting from vector-vector transmission cycle are given by $1 /\left(1-q_{v}\right)$; hence the host type reproductive number is then given by

$$
T_{1}^{h}=\frac{1}{1-q_{v}} R_{0}^{H}=R_{0}^{e}
$$

From (33), $R_{0}^{H}=R_{0}^{e}$ if $1 /\left(1-q_{v}\right)=1$; that is, $q_{v}^{*}=0$ which is the boundary condition. An important question would be, what is the proportion of vertical infection that doubles $R_{0}^{H}$ ? For $1 /\left(1-q_{v}\right)=2$ we obtain $q_{v}^{*}=0.5$, which means that if about $50 \%$ of infected mosquitoes produce infected offspring, we expect $T_{1}^{h}$ to double. Note that all secondary infections resulting from a single infected vector must occur in the next vector-host-vector transmission cycle; hence, the vector type reproductive number takes the form

$$
T_{1}^{v}=R_{0}^{H}+q_{v} .
$$

For details on the derivation of both types of reproductive numbers, see Supplementary Materials, Section A-1. A 


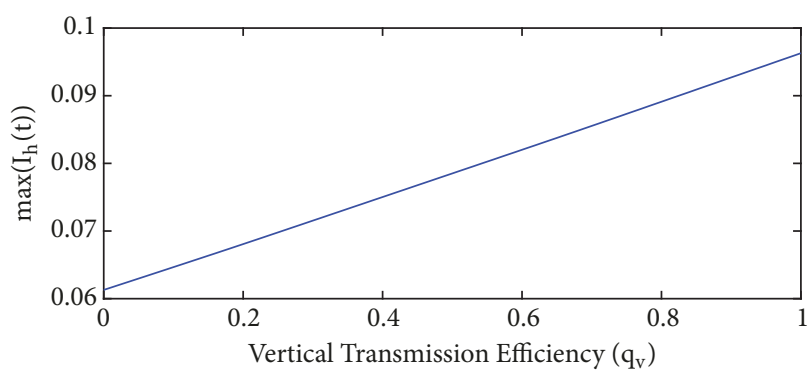

(a)

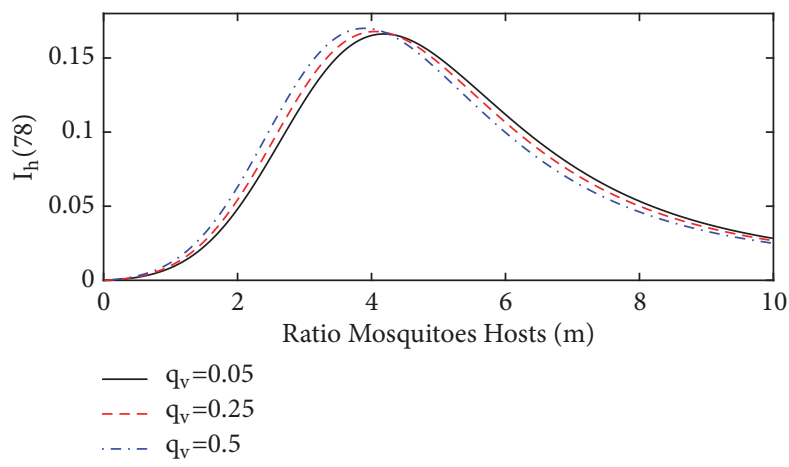

(c)

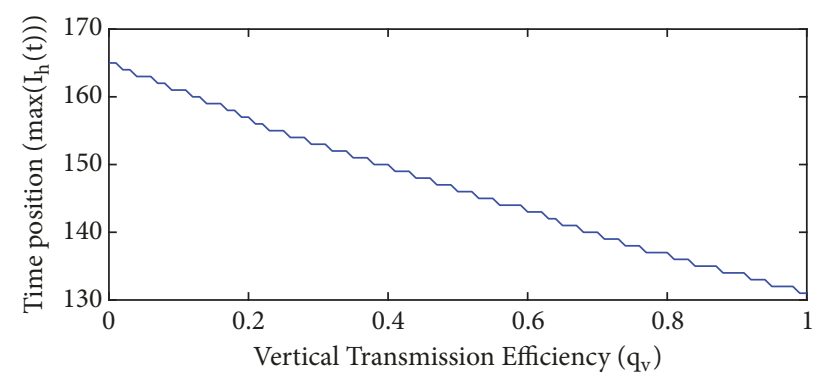

(b)

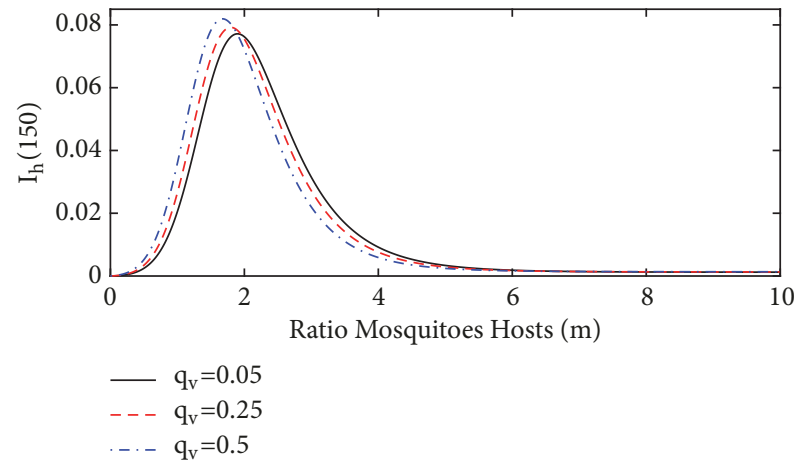

(d)

FiguRE 2: The left figure (a) explores effects of vertical transmission on the prevalence of the disease on hosts, where $\left(I_{h}\left(q_{v}\right)\right)$ is plotted at $\max \left(I_{h}(t)\right)$ for every $q_{v}$. The right figure (b) explores how the first peak of an epidemic $\left(\max \left(I_{h}(t)\right)\right)$ varies with vertical transmission efficiency $\left(q_{v}\right)$. The lower figures represent the dynamics of $I_{h}(t)$ at time position (78) corresponding to day 27 (figure c) and at time position (150) corresponding to day 50 (figure d) for different values of vertical infection.

number of methods for deriving $R_{0}$ exist and in [21] it is demonstrated that the resulting $R_{0}$ is not unique but they may have a common threshold at $R_{0}=1$. Hence, their behaviour below and above unity may show significant differences. In Figure 1๑ we examine how vertical transmission efficiency contributes to the qualitative behaviour of $R_{0}^{2}, T_{1}^{h}$, and $T_{1}^{v}$ above the unity threshold criterion. An important characteristic pattern of their relationship is that below a certain percentage of vertical transmission efficiency the three reproductive numbers are undistinguishable but beyond the relationship between vertical infection and host type reproductive number becomes exponential. This behaviour results from the fact that in each generation, the number of host infections is proportional to the number of infected mosquitoes, which decreases proportionally to the vertical infection efficiency. Hence, for low vertical infection efficiency, possibly the first generations make a significant contribution to subsequent transmission cycles (for more discussion see $[23,27])$.

In Figure 1(b) we compare the basic reproductive number $R_{0}$ derived through the next-generation method with the one derived from the endemic equilibrium $R_{0}^{e}$ with respect to vertical transmission efficiency. Clearly, the two give different relationships and it is easy to see that the effect of vertical infection is completely diluted on $R_{0}$. Hence, this measure if used for disease control effort is more likely to provide misleading guidance. The relationship of the basic reproductive number $R_{0}^{e}$ with mosquito to host ratio $m$ and vertical infection $q_{v}$ shows some interesting patterns. For $m>1$ the relationship between $R_{0}^{e}$ and $q_{v}$ is exponential as described in Figure 1(c); however for $m<1$ it is linear rendering $q_{v}$ having little effect if not negligible for $q_{v} \in(0,0.8)$ (see Figure 1(d) dashed lines). This suggests that when there are more hosts compared to mosquitoes, vertical infection efficiency becomes completely negligible as in the model settings, and if $m<1$, sustained host-vector transmissions may be impossible. On the other hand, we observe that for $q_{v} \ggg 0.8$ and even for $m<1, R_{0}^{e}$ becomes much larger highlighting the importance of the interplay between vertical infection and the ratio of mosquitoes to hosts.

Remark 7. In the settings of vector-borne diseases both host type reproductive number $T_{1}^{h}$ or $\left(R_{0}^{e}\right)$ and vector type reproductive number $T_{1}^{v}$ are critical for providing useful informative indicator for guiding disease control efforts.

\subsection{Effects of Ratio of Mosquitoes to Hosts and Vertical Trans-} mission on Disease Prevalence. To fully capture effects of vertical transmission efficiency on vector-borne transmission models, we explore how this parameter influences the disease spread patterns.

Results are shown in Figure 2. The left figure explores how the first peak of an epidemic denoted by $\max \left(I_{h}(t)\right)$ varies with vertical transmission efficiency, $q_{v}$. The results show that $\max \left(I_{h}(t)\right)$ increases linearly with $q_{v}$. The righthand figure plots the changes on time of occurrence of 
each epidemic peak at each value of vertical transmission efficiency. The results suggest that as vertical transmission efficiency increases the time position of each epidemic peak decreases linearly. This suggests that if vertical infection efficiency is increased, the first peak of an epidemic is likely to be attained earlier than the expected time. This suggests that while vertical transmission efficiency increases the epidemic size of an outbreak, it linearly decreases the time of the peak of an outbreak. The lower figures, (c) and (d), describes how the prevalence of infected hosts, $I_{h}$, varies with the ratio of female mosquitoes to hosts for different values of vertical transmission efficiency. Figure 2(c) indicates that the prevalence of the disease saturates at larger values of the ratio at earlier stage of the initial epidemic while Figure 2(d) indicates that the prevalence of the disease saturates at lower values of the ratio at later stage of the initial epidemic. This result stems from the fact that at earlier stage of the initial epidemic the size of the vector population is still very high compared to later stages of evolution of the epidemic.

\subsection{Analysis of the Model with Vector Stages and Vertical Transmission}

3.3.1. Model Equilibria. Model equilibria are obtained by setting the right-hand side of system (2) equal to zero. The model has three equilibria, two disease-free and one endemic equilibrium. Details about the derivation of the components of the endemic equilibrium are given in Section A-2 of Supplementary Materials. The first trivial equilibrium is both mosquito-free and disease-free:

$$
X^{0}=\left(L_{s}^{0}, L_{i}^{0}, S_{v}^{0}, I_{v}^{0}, S_{h}^{0}, I_{h}^{0}\right)=(0,0,0,0,1,0),
$$

which corresponds only to the presence of hosts (this can be livestock for the case of RVF). However, this equilibrium is not biologically feasible or relevant in particular in tropical regions where vector-borne diseases such as Dengue and RVF are endemic. The second equilibrium corresponds to the coexistence of both vectors and hosts but without infection. This is the disease-free equilibrium:

$$
X^{1}=\left(L_{s}^{1}, L_{i}^{1}, S_{v}^{1}, I_{v}^{1}, S_{h}^{1}, I_{h}^{1}\right)=\left(l^{*}, 0, P^{*}, 0,1,0\right),
$$

which can be used for computation of the basic reproduction number when using the next-generation method [36]. The nonzero components are

$$
\begin{gathered}
l^{*}=\frac{\delta r\left(1-Q_{0}^{-1}\right)}{\delta r+\theta+\mu_{L}}, \\
P^{*}=\frac{\theta\left(1-Q_{0}^{-1}\right)}{\theta+\delta \mu_{v}},
\end{gathered}
$$

$$
\text { with } Q_{0}=\frac{r \theta}{\mu_{v}\left(\theta+\mu_{L}\right)} \text {. }
$$

Hence, $X^{1}$ is biologically significant if $Q_{0}>1$; that is, the mosquito population exists and can establish itself if on average each adult female mosquito produces at least one female mosquito at oviposition rate $r$ during her life time period $1 / \mu_{v}$ after successfully surviving the aquatic stage at probability $\theta /\left(\theta+\mu_{L}\right)$.

The endemic equilibrium which represents the prevalence of the disease in both host and vector populations is given by

$$
X^{*}=\left(L_{s}^{*}, L_{i}^{*}, S_{v}^{*}, I_{v}^{*}, S_{h}^{*}, I_{h}^{*}\right),
$$

where

$$
\begin{aligned}
& L_{s}^{*}=l^{*}-L_{i}^{*}, \\
& S_{v}^{*}=P^{*}-I_{v}^{*}, \\
& I_{v}^{*}=\frac{\mu_{h}\left(1-S_{h}^{*}\right)}{\lambda_{h v} S_{h}^{*}}, \\
& S_{h}^{*}=1-\frac{\gamma_{h}+\mu_{h}}{\mu_{h}} I_{h}^{*}, \\
& I_{h}^{*}=\frac{\left(1-q_{v}\right) \mu_{v}\left(\gamma_{h}+\mu_{h}\right)\left(R_{0}^{c}-1\right)}{\lambda_{v h} \lambda_{h v} P^{*}\left(\gamma_{h}+\mu_{h}\right) / \mu_{h}+\lambda_{v h} /\left(1-q_{v}\right) \mu_{v}},
\end{aligned}
$$

where

$$
R_{0}^{c}=\frac{\lambda_{v h} \lambda_{h v} P^{*}}{\left(1-q_{v}\right) \mu_{v}\left(\gamma_{h}+\mu_{h}\right)} .
$$

Similar to the discussion in Section 3.1.2 the above epidemic threshold in (41) is the "effective" or "control" reproductive number since it satisfies the property that the endemic equilibrium $E^{*}$ only persists if $R_{0}^{c}$ is greater than unity and there is no backward bifurcation.

Remark 8. The relationship between the endemic equilibrium and $R_{0}$ which depends on the vector population threshold indicates that the existence of this equilibrium is governed by the norms of the vector population threshold. Therefore, this vector population threshold is an important parameter in vector control efforts as it provides a means for identifying key factors for reducing the vector population.

In the following section we derive $R_{0}$ using the nextgeneration method.

\subsubsection{The Basic Reproductive Number and Other Control} Thresholds. The basic reproductive number, $R_{0}$, is a concept of great epidemiological significance. Following the method of [36], we write system (2) considering only disease compartments, $L_{i}, I_{v}$ and $I_{h}$ as $\dot{x}=\mathscr{F}-\mathscr{V}$ with $x^{T}=\left(L_{i}, I_{v}, I_{h}\right)$, where

$$
\begin{aligned}
& \mathscr{F}=\left(\begin{array}{c}
\delta r(1-L) q_{v} I_{v} \\
\frac{\theta}{\delta}\left(1-N_{v}\right) L_{i}+\lambda_{v h} I_{h} S_{v} \\
\lambda_{h v} I_{v} S_{h}
\end{array}\right), \\
& \mathscr{V}=\left(\begin{array}{c}
\left(\theta+\mu_{L}\right) L_{i} \\
\mu_{v} I_{v} \\
\left(\gamma_{h}+\mu_{h}\right) I_{h}
\end{array}\right) .
\end{aligned}
$$


Evaluating the Jacobian of above matrices at the disease-free equilibrium $X^{1}=\left(l^{*}, 0, P^{*}, 0,1,0\right)$, the basic reproduction number is then the spectral radius of the next-generation matrix; that is, $R_{0}=\rho\left(\mathscr{F}\left(X^{1}\right) \mathscr{V}\left(X^{1}\right)^{-1}\right)$,

$$
R_{0}=\frac{1}{2} q_{v}+\frac{1}{2} \sqrt{q_{v}^{2}+4 R_{0}^{H}} \text {, with } R_{0}^{H}=\frac{\lambda_{v h} \lambda_{h v} P^{*}}{\mu_{v}\left(\gamma_{h}+\mu_{h}\right)}
$$

Following the discussion in Section 3.1.2 this epidemic threshold (43) can lead to an underestimate of the control effort required if used to guide disease control strategies. To overcome this inconsistency we use type reproductive numbers to estimate efforts required to provide informative indicators in control of vector-borne diseases. To avoid repetition we omit the steps required in the derivation process, such that the host type reproductive number is

$$
T_{1}^{h}=\frac{R_{0}^{H}}{1-q_{v}}=R_{0}^{c},
$$

and the vector type reproductive number is then given by

$$
T_{1}^{v}=R_{0}^{H}+q_{v}
$$

In the majority of mosquito-borne diseases, treatment or adequate vaccine is not likely to be feasible, either due to financial constraints or nonavailability, in particular in African communities with low resilience to economic challenges. In such cases the main preventive measures are individual protection against mosquito bites and the control of the proliferation of both the larval and adult mosquitoes. This can be achieved by lowering appropriate epidemic thresholds below unity, that is, forcing the reproductive number of the pathogen below 1 . In the context of our model this implies reducing either of or both type reproductive numbers $\left(T_{1}^{h}\right.$ and/or $T_{1}^{v}$ ) below unity. Hence, we define the eradication effort to be the percentage reduction in the mosquito population size required to prevent endemic transmission of the pathogen [23], such that if using $T_{1}^{h}$ the eradication effort is $100\left(1-1 / T_{1}^{h}\right)=100\left(R_{0}^{H}+q_{v}-\right.$ 1) $/ R_{0}^{H}$ and for $T_{1}^{v}$ the effort required to eradicate the disease is $100\left(1-1 / T_{1}^{v}\right)=100\left(R_{0}^{H}+q_{v}-1\right) /\left(R_{0}^{H}+q_{v}\right)$. Therefore, vertical infection at $q_{v} \%$ can be responsible for at most $q_{v} \%$ of the required eradication effort, provided $R_{0}^{H}>1$.

3.3.3. Stability of the Host-Only System. This fixed point $X^{0}=$ $\left(L_{s}^{0}, L_{i}^{0}, S_{v}^{0}, I_{v}^{0}, S_{h}^{0}, I_{h}^{0}\right)=(0,0,0,0,1,0)$ is an equilibrium point of the host-only system given by

$$
\frac{d S_{h}}{d t}=\mu_{h}\left(1-S_{h}\right)
$$

which is local and globally asymptotically stable for all $t>0$ as the derivative $\left(\mu_{h}\left(1-S_{h}\right)\right)^{\prime}$ is negative.

3.3.4. Local Stability of the Disease-Free Equilibrium. The local stability of the disease-free equilibrium point $X^{1}=$ $\left(l^{*}, 0, P^{*}, 0,1,0\right)$ is established by analysing the eigenvalues of the Jacobian matrix at $X^{1}$ :

$$
J\left(E^{1}\right)=\left(\begin{array}{cccccc}
-\delta r P^{*}-\left(\theta+\mu_{L}\right) & \delta r P^{*} & \delta r\left(1-l^{*}\right) & \delta r\left(1-l^{*}\right)\left(1-q_{v}\right) & 0 & 0 \\
0 & -\left(\theta+\mu_{L}\right) & 0 & \delta r\left(1-l^{*}\right) q_{v} & 0 & 0 \\
\frac{\theta}{\delta}\left(1-P^{*}\right) & 0 & -\frac{\theta}{\delta} l^{*}-\mu_{v} & -\frac{\theta}{\delta} l^{*} & 0 & -\lambda_{v h} P^{*} \\
0 & \frac{\theta}{\delta}\left(1-P^{*}\right) & 0 & -\mu_{v} & 0 & \lambda_{v h} P^{*} \\
0 & 0 & 0 & -\lambda_{h v} & -\mu_{h} & 0 \\
0 & 0 & 0 & \lambda_{h v} & 0 & -\left(\gamma_{h}+\mu_{h}\right)
\end{array}\right) .
$$

Direct computation shows that $\lambda_{1}=-\mu_{h}$ is an eigenvalue of matrix (47) and the remaining are solutions of equation

$$
\lambda^{5}+c_{4} \lambda^{4}+c_{3} \lambda^{3}+c_{2} \lambda^{2}+c_{1} \lambda+c_{0}=0
$$

where

$$
\begin{aligned}
c_{4}= & \delta r P^{*}+2\left(\theta+\mu_{L}\right)+\left(\gamma_{h}+\mu_{h}\right)+\frac{\theta}{\delta} l^{*}+2 \mu_{v}>0, \\
c_{3}= & \left(1-q_{v}\right)\left(\gamma_{h}+\mu_{h}\right) \mu_{v}\left(1-R_{0}^{c}\right)+\delta r P^{*}\left(\gamma_{h}+\mu_{h}\right. \\
& \left.+\left(\frac{\theta l^{*}}{\delta}\right)+2 \mu_{v}\right)+\left(\theta+\mu_{L}+\mu_{v}\right)^{2}+\frac{\theta}{\delta} \\
& \cdot l^{*}\left(2\left(\theta+\mu_{L}\right)+\gamma_{h}+\mu_{h}+\mu_{v}\right)+2\left(\gamma_{h}+\mu_{h}\right)(\theta \\
& \left.+\mu_{L}+\mu_{v}\right)+\left(1-q_{v}\right) \mu_{v}\left(\theta+\mu_{L}-\left(\gamma_{h}+\mu_{h}\right)\right)>0,
\end{aligned}
$$

$$
\begin{aligned}
c_{2} & =\left(1-q_{v}\right)\left(\gamma_{h}+\mu_{h}\right) \mu_{v}\left(1-R_{0}^{c}\right)\left(\delta r P^{*}+2\left(\theta+\mu_{L}\right)\right. \\
& \left.+\left(\frac{\theta l^{*}}{\delta}\right)+\mu_{v}\right)+\left(1-q_{v}\right) \mu_{v}\left(\theta+\mu_{L}-\left(\gamma_{h}+\mu_{h}\right)\right) \\
& +\left(\delta r P^{*}+\theta+\mu_{L}+\left(\frac{\theta l^{*}}{\delta}\right)+\mu_{v}\right)+\left(\gamma_{h}+\mu_{h}\right)(\theta \\
& \left.+\mu_{L}+\mu_{v}\right)^{2} \\
& +\delta r P^{*}\left[\left(\theta+\mu_{L}\right)\left(\gamma_{h}+\mu_{h}+\left(\frac{\theta l^{*}}{\delta}\right)+\mu_{v}\right)\right. \\
& \left.+\left(\gamma_{h}+\mu_{h}\right)\left(\left(\frac{\theta l^{*}}{\delta}\right)+2 \mu_{v}\right)+\mu_{v}\left(\left(\frac{\theta l^{*}}{\delta}\right)+\mu_{v}\right)\right]
\end{aligned}
$$




$$
\begin{aligned}
& +\frac{\theta}{\delta} l^{*}\left(\theta+\mu_{L}\right)\left(\theta+\mu_{L}+2\left(\gamma_{h}+\mu_{h}\right)+\mu_{v}\right)+\frac{\theta}{\delta} \\
\cdot & l^{*}\left(\gamma_{h}+\mu_{h}\right) \mu_{v}>0, \\
c_{1}= & \left(1-q_{v}\right)\left(\gamma_{h}+\mu_{h}\right) \mu_{v}\left(1-R_{0}^{c}\right) \\
\cdot & {\left[\delta r P^{*}\left(\theta+\mu_{L}+\left(\frac{\theta l^{*}}{\delta}\right)+\mu_{v}\right)+\left(\theta+\mu_{L}\right)^{2}\right.} \\
+ & \left.\left(\theta+\mu_{L}\right) \mu_{v}+2\left(\theta+\mu_{L}\right)\left(\frac{\theta l^{*}}{\delta}\right)\right] \\
+ & {\left[\delta r P^{*}\left(\left(\frac{\theta l^{*}}{\delta}\right)+\mu_{v}\right)+\left(\theta+\mu_{L}\right)\left(\frac{\theta l^{*}}{\delta}\right)\right) } \\
\cdot & {\left[\left(1-q_{v}\right) \mu_{v}\left(\theta+\mu_{L}-\left(\gamma_{h}+\mu_{h}\right)\right)+\left(\gamma_{h}+\mu_{h}\right) \mu_{v}\right] } \\
+ & \left(\theta+\mu_{L}\right)\left(\gamma_{h}+\mu_{h}\right)\left(\frac{\theta l^{*}}{\delta}\right)\left(\delta r P^{*}+\left(\theta+\mu_{L}\right)\right)>0, \\
c_{0}= & \left(1-q_{v}\right)\left(\theta+\mu_{L}\right)\left(\gamma_{h}+\mu_{h}\right) \mu_{v}\left(1-R_{0}^{c}\right) \\
& \cdot\left[\delta r P^{*}\left(\left(\frac{\theta l^{*}}{\delta}\right)+\mu_{v}\right)+\left(\frac{\theta l^{*}}{\delta}\right) \mu_{v}\right] .
\end{aligned}
$$

By Descartes' rule of signs, since $c_{4}>0, c_{3}>0, c_{2}>0$, and $c_{1}>0$, then all roots of (48) are negative or have a negative real part for $c_{0}>0$. The coefficient $c_{0}$ is nonnegative if and only if $R_{0}^{c}<1$; hence the following result holds.

Theorem 9. The disease-free equilibrium point $X^{1}=\left(l^{*}, 0\right.$, $\left.P^{*}, 0,1,0\right)$ is locally asymptotically stable whenever $R_{0}^{c}<1$.

3.3.5. Global Stability of the Disease-Free Equilibrium. To establish the global asymptotic stability (GAS) of $X^{1}$ we use results obtained by Kamgang and Sallet [40], which are the extension of some results in [36]. Around the disease-free equilibrium system (2) can be written as

$$
\begin{aligned}
& \dot{x}_{S}=A_{1}(x)\left(x_{S}-x_{E^{1}, S}\right)+A_{12}(x) x_{I} \\
& \dot{x}_{I}=A_{2}(x) x_{I}
\end{aligned}
$$

where $x_{S}$ is the vector representing disease-free compartments $\left(L_{s}, S_{v}, S_{h}\right)$ and the vector $x_{I}$ represents the state of infected compartments $\left(L_{i}, I_{v}, I_{h}\right)$. This requires rewriting system equation around $X^{1}$, as follows:

$$
\begin{aligned}
\frac{d S_{h}}{d t}= & \mu_{h}-\lambda_{h v} I_{v} S_{h}-\mu_{h} S_{h} \\
= & -\mu_{h}\left(S_{h}-S_{h}^{1}\right)-\lambda_{h v} I_{v} S_{h} \\
\frac{d L_{s}}{d t}= & \delta r\left(1-L_{s}\right)\left(S_{v}+\left(1-q_{v}\right) I_{v}\right) \\
& -\delta r L_{i}\left(S_{v}+\left(1-q_{v}\right) I_{v}\right)-\left(\theta+\mu_{L}\right) L_{s} \\
= & -\left(\delta r S_{v}+\theta+\mu_{L}\right)\left(L_{s}-L_{s}^{1}\right) \\
& +\delta r\left(1-L_{s}^{1}\right)\left(S_{v}-S_{v}^{1}\right)-\delta r S_{v} L_{i} \\
& +\delta r\left(1-q_{v}\right)(1-L) I_{v} .
\end{aligned}
$$

Note that the latter arises from $\left(\theta+\mu_{L}\right) L_{s}^{1}=\delta r\left(1-L_{s}^{1}\right) S_{v}^{1}$. Then, the following matrices are obtained,

$$
\begin{aligned}
& A_{1}(x)=\left(\begin{array}{ccc}
-\left(\delta r S_{v}+\theta+\mu_{L}\right) & \delta r\left(1-L_{s}^{1}\right) & 0 \\
\frac{\theta}{\delta}\left(1-N_{v}\right) & -\mu_{v} & 0 \\
0 & 0 & -\mu_{h}
\end{array}\right), \\
& A_{12}(x)=\left(\begin{array}{ccc}
-\delta r S_{v} & \delta r\left(1-q_{v}\right)(1-L) & 0 \\
0 & 0 & -\lambda_{v h} S_{v} \\
0 & -\lambda_{v h} S_{v} & 0
\end{array}\right),
\end{aligned}
$$

$$
\begin{aligned}
& A_{2}(x) \\
& \quad=\left(\begin{array}{ccc}
-\left(\theta+\mu_{L}\right) & \delta r(1-L) q_{v} & 0 \\
\frac{\theta}{\delta}\left(1-N_{v}\right) & -\mu_{v} & \lambda_{v h} S_{v} \\
0 & \lambda_{h v} & -\left(\gamma_{h}+\mu_{h}\right)
\end{array}\right) .
\end{aligned}
$$

From basic matrix properties and direct algebraic computation it follows that all eigenvalues of matrix $A_{1}$ are real and negative and both $A_{1}$ and $A_{2}$ are Metzler matrices. Hence, system $\dot{x}_{S}=A_{1}(x)\left(x_{S}-x_{X^{1}, S}\right)$ is GAS at the disease-free equilibrium $x_{X^{1}, s}$. To establish the global stability of overall system (50) at $X^{1}$ conditions of the following theorem in [40] must be satisfied.

Theorem 10. Let $\Phi \subset \mathcal{U}=\mathbb{R}_{+}^{3} \times \mathbb{R}_{+}^{3}$. System (50) is of class $C^{1}$, defined on $\mathcal{U}$ if

(1) $U$ is positively invariant relative to (50).

(2) The system $\dot{x}_{S}=A_{1}(x)\left(x_{S}-x_{D F E, S}\right)$ is GAS at $x_{D F E, S}$.

(3) For any $x \in \Phi$, matrix $A_{2}(x)$ is Metzler irreducible.

(4) There exists a matrix $\bar{A}_{2}$, which is an upper bound of the set $\mathscr{M}=\left\{A_{2}(x) \in \mathscr{M}_{3}(\mathbb{R}) \mid x \in \bar{\Phi}\right\}$, with the property that if $\bar{A}_{2} \in \mathscr{M}$, for any $\bar{x} \in \bar{\Phi}$, such that $A_{2}(\bar{x})=\bar{A}_{2}$, then $\bar{x} \in \mathbb{R}^{3} \times\{0\}$.

(5) The stability modulus of $\bar{A}_{2}, \alpha\left(\bar{A}_{2}\right)=\max _{\lambda \in S_{p}\left(A_{2}\right)} \operatorname{Re}(\lambda)$, satisfies $\alpha\left(\bar{A}_{2}\right) \leq 0$.

Then, $X^{1}$ is GAS in $\bar{\Phi}$.

Proof. Clearly, conditions (1)-(3) of the theorem have been satisfied. For all $x \in \Phi, A_{2}(x)$ is irreducible because $(I+$ $\left.\left|A_{2}(x)\right|\right)^{2}>0$. An upper bound of the set of matrices $\mathscr{M}$, which is the matrix $\bar{A}_{2}$, is given by matrix $A_{2}(\bar{x})$, where $\bar{x}=$ $\left(\bar{L}_{s}^{1}, 0, \bar{S}_{v}^{1}, 0,1,0\right) \in \mathbb{R}^{3} \times\{0\}$, with $\bar{L}_{s}^{1}=K_{1}$ and $\bar{S}_{v}^{1}=K_{2}$. Similarly matrix $\bar{A}_{2}$ is irreducible. Recall that from the PerronFrobenius theorem for an irreducible matrix you get that one of the matrix eigenvalues is positive and greater than or equal to all others, that is, the dominant eigenvalue. Matrix $A_{2}$ is exactly the matrix used to compute the basic reproductive number, i.e., the dominant eigenvalue. For more details or proof in general settings see [40].

Now conditions (1)-(4) have been verified. To check the last condition, we make use of the following Lemma [40]. 
Lemma 11. Let $H$ be a square Metzler matrix written in block form $H=\left(\begin{array}{ll}A & B \\ C & D\end{array}\right)$, with $A$ and $D$ square matrices. $H$ is Metzler stable if and only if matrices $A$ and $D-C A^{-1} B$ are Metzler stable.

Matrix $A_{2}(x)$ in block matrices takes the following components:

$$
\begin{aligned}
A & =\left(-\left(\theta+\mu_{L}\right)\right), \\
B & =\left(\begin{array}{ll}
\delta r(1-L) q_{v} & 0
\end{array}\right), \\
C & =\left(\begin{array}{ll}
\frac{\theta}{\delta}\left(1-N_{v}\right) & 0
\end{array}\right)^{T}, \\
D & =\left(\begin{array}{ll}
-\mu_{v} & \lambda_{v h} P^{*} \\
\lambda_{h v} & -\left(\gamma_{h}+\mu_{h}\right)
\end{array}\right) .
\end{aligned}
$$

Clearly, $A$ is a Metzler stable matrix and

$$
D-C A^{-1} B=\left(\begin{array}{cc}
-\mu_{v}\left(1-q_{v}\right) & \lambda_{v h} P^{*} \\
\lambda_{h v} & -\left(\gamma_{h}+\mu_{h}\right)
\end{array}\right)
$$

is also Metzler stable if $\mu_{v}\left(1-q_{v}\right)\left(\gamma_{h}+\mu_{h}\right)-\lambda_{h v} \lambda_{v h} P^{*} \geqslant 0$, that is, $1-R_{0}^{c} \geqslant 0$. Therefore, from Theorem 10 and Lemma 11 the following result holds.

Theorem 12. For $Q_{0}>1$ there exists a unique diseasefree equilibrium $X^{1}=\left(l^{*}, 0, P^{*}, 0,1,0\right)$, which is globally asymptotically stable whenever $R_{0}^{c} \leqslant 1$.

Remark 13. The above result is of great epidemiological importance. It highlights two fundamental indicators: (1)

Although vector control is central for control of vector-borne diseases, it does not mean eliminating all the vectors. Note that the effect of this include both infected and noninfected mosquitoes. (2) Disease eradication efforts are independent of the initial sizes of both vector and host populations; however, the ratio between the two populations is a key factor.

3.3.6. Local Stability of the Endemic Equilibrium. Results of the local stability of the disease-free equilibrium $X^{1}$ suggest that for $R_{0}^{c}=1$ the Jacobian matrix (47) has zero eigenvalue while the remaining eigenvalues are negative or have a negative real part. Since the algebraic computation involved when establishing the stability of the endemic equilibrium through linearization is quite extensive, we employ the centre manifold theory [41]. This theory is used to examine existence of backward or forward bifurcation. The bifurcation occurs at $R_{0}^{c}=1$, and choosing $q_{v}=q_{v}^{*}$ as a bifurcation parameter, then

$$
q_{v}^{*}=1-\frac{\lambda_{h v} \lambda_{v h} P^{*}}{\mu_{v}\left(\gamma_{h}+\mu_{h}\right)}, \Longrightarrow R_{0}^{H} \leqslant 1 .
$$

The Jacobian matrix $J\left(X^{1}, q_{v}^{*}\right)$ is the same as matrix (47); hence, $J\left(X^{1}, q_{v}^{*}\right)$ has a simple zero eigenvalue $\lambda=0$ when $R_{0}^{c}=1$ and others given as $\lambda=-\mu_{h}$ and roots of the polynomial $\lambda^{4}+c_{4} \lambda^{3}+c_{3} \lambda^{2}+c_{2} \lambda+c_{1}=0$, where $c_{1}, c_{2}, c_{3}, c_{4}$ are as defined in Section 3.3.4. Let $\omega=\left(\omega_{1}, \omega_{2}, \omega_{3}, \omega_{4}, \omega_{5}, \omega_{6}\right)$ be the right eigenvector associated with zero eigenvalue of the Jacobian matrix $J\left(X^{1}, q_{v}^{*}\right)$. Its components are derived by solving $J\left(X^{1}, q_{v}^{*}\right) \times \omega=0$, which gives

$$
\omega=\left(F^{*} \omega_{4}, H^{*} \omega_{4}, G^{*} \omega_{4}, \omega_{4},-\frac{\lambda_{h v}}{\mu_{h}} \omega_{4}, \frac{\lambda_{h v}}{\gamma_{h}+\mu_{h}}\right)
$$

where

$$
\begin{aligned}
F^{*} & =\frac{\left[r \theta \mu_{h}\left(Q_{0}^{-1}-q_{v}^{*}\right)+\left(1-q_{v}^{*}\right) \mu_{h}\left(\gamma_{h}+\mu_{h}\right)\left(\theta+\mu_{L}\right)\right] \delta r\left(\theta+\delta \mu_{v}\right)}{r \theta^{2} \mu_{h}\left(1-Q_{0}^{-1}\right)\left(\delta r+\theta+\mu_{L}\right)} \\
G^{*} & =\frac{\left(1-q_{v}^{*}\right) \mu_{v} \theta\left(\theta+\mu_{L}\right)\left[\left(\gamma_{h}+\mu_{h}\right)\left(\delta r+\theta+\mu_{L}\right)-\mu_{h}\left(\theta+\mu_{L}\right)\left(Q_{0}^{v}-1\right)\right]+\mu_{h} \mu_{v}\left(\theta+\mu_{L}\right)^{2}\left[\theta+\delta \mu_{v}\left(1-Q_{0} q_{v}^{*}\right)\right]}{r \theta\left(\theta+\delta \mu_{v}\right)\left(1-Q_{0}^{-1}\right)} \\
H^{*} & =\frac{\delta r q_{v}^{*}\left(\theta+\delta \mu_{v}\right)}{\theta\left(\delta r+\theta+\mu_{L}\right)} \\
Q_{0}^{v} & =\frac{r \theta}{\left(1-q_{v}^{*}\right) \mu_{v}\left(\theta+\mu_{L}\right)} .
\end{aligned}
$$

Clearly, $F^{*}$ and $G^{*}$ are nonnegative if and only if $q_{v}^{*} \leqslant 1 / Q_{0}$, $Q_{0}^{v}>1$, and $Q_{0}>1$. Note that $Q_{0}^{v}$ gives the average number of emerged infected female mosquitoes produced by one infected female mosquito. Hence, for $Q_{0}^{v}>1$ infected mosquitoes will persist in the population, leading to disease persistence. Let us denote $v=\left(v_{1}, v_{2}, v_{3}, v_{4}, v_{5}, v_{6}\right)$ as the left eigenvector associated with zero eigenvalue of the Jacobian matrix $J\left(X^{1}, q_{v}^{*}\right)$. Its components are obtained when solving $\left[J\left(X^{1}, q_{v}^{*}\right)\right]^{T} \times \omega=0,(T$ represents transpose $)$, which gives

$$
v=\left(0, M^{*} v_{4}, 0, v_{4}, 0, \frac{\lambda_{v h} P^{*}}{\gamma_{h}+\mu_{h}} v_{4}\right)
$$

where $M^{*}=\theta \mu_{v}\left(\delta r+\theta+\mu_{L}\right) / \delta r\left(\theta+\mu_{L}\right)\left(\theta+\delta \mu_{v}\right)$. 
TABLE 1: Existence and stability of model equilibria.

\begin{tabular}{|c|c|c|c|c|}
\hline & & $X^{0}$ & $X^{1}$ if $\left(0<q_{v}<1\right)$ & $X^{*}$ if $\left(0<q_{v}<1\right)$ \\
\hline I. & $Q_{0}<1 \& R_{0}^{c}<1$ & GS & DNE & DNE \\
\hline II. & $Q_{0}>1 \& R_{0}^{c}<1$ & DNE & GS & DNE \\
\hline III. & $Q_{0}>1 \& R_{0}^{c}>1$ & DNE & US & LS \\
\hline IV. & $Q_{0}<1 \& R_{0}^{c}>1$ & US & DNE & DNE \\
\hline
\end{tabular}

DNE: does not exist, US: unstable, LS: locally stable, GS: globally stable.

Computation of Bifurcation Parameters $a^{*}$ and $b^{*}$. Because $v_{1}=v_{3}=v_{5}=0$ the associated nonzero partial derivatives of $f_{1}, f_{3}, f_{5}$ are not considered and the remaining nonzero partial derivatives of system $f=\left(f_{1}, f_{2}, f_{3}, f_{4}, f_{5}, f_{6}\right)$ at $\left(X^{1}, q_{v}^{*}\right)$ are given by $\partial^{2} f_{2} / \partial x_{1} \partial x_{4}=\partial^{2} f_{2} / \partial x_{2} \partial x_{4}=-\delta r q_{v}^{*}$, $\partial^{2} f_{4} / \partial x_{2} \partial x_{4}=\partial^{2} f_{4} / \partial x_{3} \partial x_{2}=-\theta / \delta, \partial^{2} f_{4} / \partial x_{3} \partial x_{6}=\lambda_{v h}$, and $\partial^{2} f_{6} / \partial x_{4} \partial x_{5}=\lambda_{h v}$.

Thus, $a^{*}=2 v_{2} \omega_{1} \omega_{4}\left(\partial^{2} f_{2} / \partial x_{1} \partial x_{4}\right)+2 v_{2} \omega_{2} \omega_{4}\left(\partial^{2} f_{2} /\right.$ $\left.\partial x_{2} \partial x_{4}\right)+2 v_{4} \omega_{2} \omega_{4}\left(\partial^{2} f_{4} / \partial x_{2} \partial x_{4}\right)+2 v_{4} \omega_{3} \omega_{2}\left(\partial^{2} f_{4} / \partial x_{3} \partial x_{2}\right)+$ $2 v_{4} \omega_{3} \omega_{6}\left(\partial^{2} f_{4} / \partial x_{3} \partial x_{6}\right)+2 v_{6} \omega_{4} \omega_{5}\left(\partial^{2} f_{6} / \partial x_{4} \partial x_{5}\right)$, which after some algebraic simplification gives

$$
\begin{aligned}
a^{*} & =-2 v_{4} \omega_{4}^{2}\left(\delta r q_{v}^{*} F^{*} M^{*}+\delta r q_{v}^{*} H^{*} M^{*}+\frac{\theta}{\delta} H^{*}\right. \\
& \left.+G^{*} H^{*}+\left(1-q_{v}^{*}\right) \mu_{v}\left(\frac{\lambda_{v h}}{\mu_{h}}-\frac{G^{*}}{P^{*}}\right)\right) .
\end{aligned}
$$

Given that the vital dynamics of the host are much more slower $\lambda_{v h} / \mu_{h} \ggg G^{*} / P^{*}$ and using the property that $v \omega=1$, then $a^{*}<0$ for $\omega_{4}>0$. For $b^{*}$ the nonzero partial derivative of $f=\left(f_{1}, f_{2}, f_{3}, f_{4}, f_{5}, f_{6}\right)$ at $\left(X^{1}, q_{v}^{*}\right)$ is $\partial^{2} f_{2} / \partial x_{4} \partial q_{v}^{*}=$ $\delta r\left(1-l^{*}\right)$, such that $b^{*}=v_{2} \omega_{4}\left(\partial^{2} f_{2} / \partial x_{4} \partial q_{v}^{*}\right)=\mu_{v} v_{2} \omega_{4}$. Using the property that $v \omega=1$ we obtain that $b^{*}>0$. Since $a^{*}<0$ for $\omega_{4}>0$ and $b^{*}>0$, the model (2) exhibits a forward bifurcation at $R_{0}^{c}=1$. Therefore, the following result holds.

Theorem 14. The endemic equilibrium point $X^{*}=\left(L_{s}^{*}, L_{i}^{*}\right.$, $\left.S_{v}^{*}, I_{v}^{*}, S_{h}^{*}, I_{h}^{*}\right)$ exists and is locally asymptotically stable for $R_{0}^{c}>$ 1 .

Remark 15. The condition $Q_{0}^{v}>1$ is an indication that vertical transmission is vital for disease long-term persistence. A particular example is the case of Dengue fever and RVF. Upon failure of this condition, it is much more likely that results of Theorem 14 do not hold. Another important feature of $Q_{0}^{v}$ is that vertical infection is proportional to $Q_{0}$, and hence vector control is still a viable control strategy even for vector-borne disease with transovarial transmission.

The above stability results and those in Theorems 9, 10, 12, and 14 can be summarized in Table 1 and in the bifurcation diagram in Figure 3.

3.4. Numerical Results of the Model with Vector Stages and Vertical Transmission. Numerical analysis using reasonable parameter values for RVF is carried out. In Figure 4 we plot time series of infected larval, adult mosquitoes and hosts, and their respective phase portraits. The latter are plotted

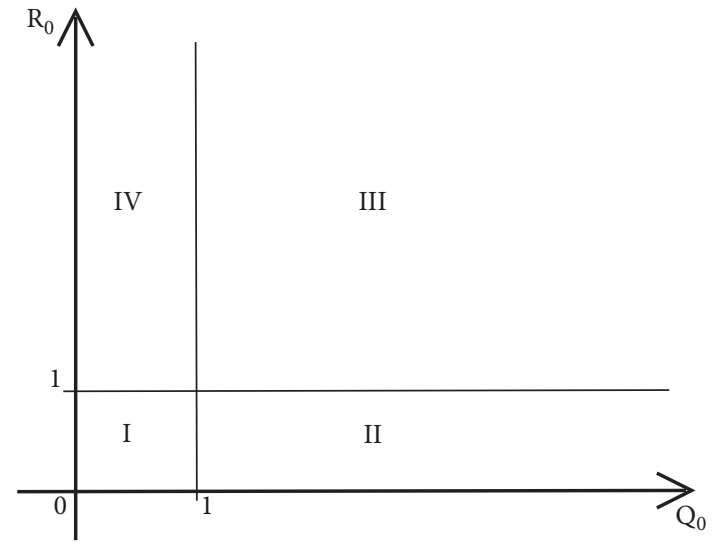

FIGURE 3: The bifurcation diagram in $\left(Q_{0}, R_{0}^{c}\right)$ plane. The stability of equilibria in each region is given in Table 1.

for $t \in\left[\begin{array}{ll}0 & 50\end{array}\right]$ years for observing long-term dynamics. The parameter values used give $R_{0}^{c}>1$ and existence of a stable endemic equilibrium. These results not only confirm findings by analytical analysis (Theorems 9 and 12) but suggest that this equilibrium is also global asymptotic stable for the chosen parameter values. This also implies that the endemic equilibrium is stable not only close to the bifurcation point but also for $R_{0}^{c}$ beyond unity (see inner figures, Figures $4(a)-4(c))$.

\section{Discussion and Conclusion}

One of the main contributions of mathematical epidemic models is that they enhance our understanding of disease transmission and public health planning. This is possible because with models we are able to derive critical epidemic thresholds in terms of model parameters which carry important disease features and key players. One of these measures is the basic reproduction number $R_{0}$, which if greater than unity implies that the disease will persist in the population and fade out otherwise. However, a major challenge is to derive the correct $R_{0}$ for disease models in which infection transmission is intermediated by another host. This is the case of vector-borne diseases, where practical methods such as the next-generation matrix for deriving $R_{0}$ fails to give the correct value [21]. Rather, it gives the geometric mean of secondary infection per generation. Using the basic model we discussed alternative measures, known as host and vector type reproductive numbers $\left(T_{1}^{h}\right.$ and $T_{1}^{v}$, respectively) $[38,39]$, to be used for guiding disease control efforts. Further, for 


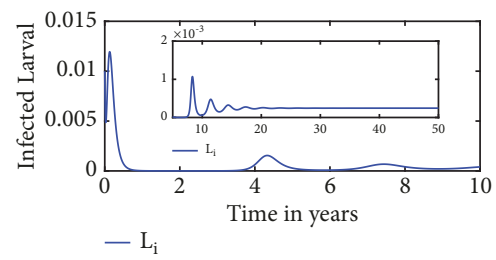

(a)

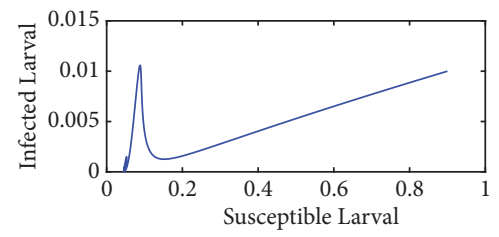

(d)

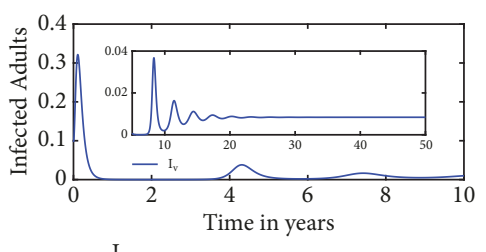

(b)

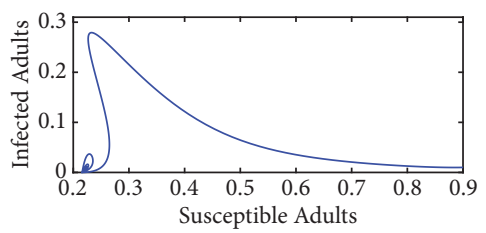

(e)

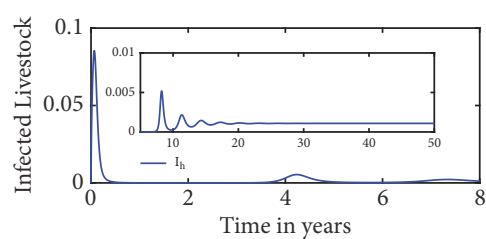

$-\mathrm{I}_{\mathrm{h}}$

(c)

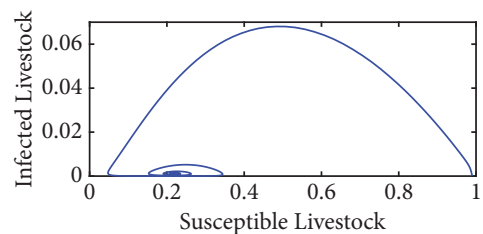

(f)

Figure 4: Time series and phase diagrams plot. The inner figures describe the temporal evolution of $L_{i}, I_{v}, I_{h}$ after the transient period for $R_{0}^{c}=2.1002$, while for the outer figures $R_{0}^{c}=1.0012$. Parameter values used for $R_{0}^{c}=1.0012$ are $\mu_{v}=1 / 25, \mu_{h}=1 /(8 * 360)$, $p_{h v}=0.14, p_{v h}=$ $0.35, \alpha=256 / 360, \gamma_{h}=1 / 4, m=1.5, q_{v}=0.2, \theta=1 / 10, \mu_{L}=1 / 18, r=1 / 12, \delta=0.4$ and parameter values used for $R_{0}^{c}=2.1002$ are given in Table 1 of Section A-3 of Supplementary Materials.

both the basic and the complex model we showed that the basic reproductive number derived from the existence of endemic equilibrium is the correct epidemic measure and it is equivalent to the host type reproductive number $T_{1}^{h}$. In fact, this is an alternative method for finding $R_{0}$ when it is possible to derive the explicit components of the endemic equilibrium point $[21,42]$. Then we established important relationships between the reproductive numbers $R_{0}^{2}, T_{1}^{h}, T_{1}^{v}$ and vertical infection efficiency. It follows that there is a critical threshold of vertical infection from which the contribution of this transmission route becomes significant. Otherwise, if vertical infection efficiency is very low, its contribution to disease persistence may be insignificant. The work in $[23,27]$ discusses this feature in more detail and their findings suggest that these results stem from the fact that for low vertical infection efficiency the virus is rapidly lost unless there is a regular disease amplification in the host population. Recent entomological studies have confirmed this situation in particular for RVF where there has been more and more evidence of disease interepidemic activities [7, 1720], underlined by sporadic outbreaks on livestock at low levels. During this period vertical infection plays a key role for providing protection against chance extinction, but only if it is efficient enough to span several mosquito generations [23], since at the end of unfavourable season the horizontally transmitted epidemic is declining.

Also, our results demonstrated that while the relationship between $R_{0}$ and vertical infection efficiency is linear, the relationship between the correct threshold $R_{0}^{e}$ and vertical infection efficiency is exponential. This shows that the failure of $R_{0}$ as a critical epidemic threshold compounds itself when varying vertical transmission. Therefore, more caution is needed when deriving the basic reproductive number in vector-borne disease systems that include vertical transmission as one of the transmission modes. Another important property to highlight in our models is the female mosquitoes to host ratio which is more likely to be responsible for the asymmetric relationship between the host-to-vector and vector-to-host reproductive numbers as discussed in other studies $[28,43]$. We found that in the presence of vertical infection the critical ratio of female mosquitoes to hosts decreases linearly when increasing vertical transmission efficiency. This result indicates an earlier occurrence of the point where an epidemic is just possible as above this level the equilibrium prevalence is expected to rapidly increase to its asymptotic value [37]. Further we analysed how vertical infection affects the prevalence of the disease at the first peak of an epidemic. The results showed that the prevalence at the first peak is positively correlated to vertical transmission efficiency while the time position of occurrence of each peak is negatively correlated to vertical transmission efficiency. These findings suggest that while vertical transmission efficiency increases the epidemic size of an outbreak, it reduces the duration of an outbreak. Additionally, the relationship between the prevalence of the disease and ratio of female mosquitoes to hosts was accessed for different values of vertical infection. Analysis showed that there is an optimal value of the ratio above which the prevalence of the disease decreases. However, such optimal value changes according to the stage of epidemic development, such that at early stage of the epidemic the optimal value is attained much later than that at later stage of the epidemic. Another interesting result is that after the optimal value of the ratio the effect of vertical transmission changes where higher values have less effect compared to lower values of vertical transmission efficiency.

Extending the basic model to include larval compartments with maximal capacities for both larval and adult populations we derived important critical thresholds for both vector population and disease system. We found that the model exhibits three boundary equilibria, namely, a mosquito-free system, a mosquito-host system but diseasefree, and one where the disease persists in both populations. 
A detailed stability analysis is presented and numerical simulations are conducted using parameter values relevant to Rift Valley fever. The mathematical results are used to interpret the biological implications of the relationships between $R_{0}$, vertical infection, and the ratio of female mosquitoes to hosts for assessing viable control measures. Our analysis shows that the qualitative behaviours of the system are completely determined by three key quantities: $Q_{0}$, the vector population threshold; $Q_{0}^{v}$, the average number of female mosquitoes produced by a single infected mosquito; and $R_{0}^{c}$, the effective reproductive number (see the bifurcation diagram in Figure 3 and Table 1). These results provide important qualitative understanding of the interaction between vertical infection and ratio of female mosquitoes to hosts on the prevalence of the disease.

The mosquito population persists if each female adult mosquito produces at least one larval mosquito after surviving the larval stage. Then, if an infected individual is introduced in the community without any form of protection, the disease will invade the system leading to appearance of an endemic equilibrium if the epidemic criterion is satisfied $\left(R_{0}^{e}>1\right)$. The biological implication of this result is that the disease can only invade an area if already colonized by the mosquito population as expected. Hence, measures targeting critical features of the life cycle of the vector are viable disease control strategies. Furthermore, we found that the persistence of the disease is subjected to vertical transmission efficiency governed by the epidemic criterion $Q_{0}^{v}>1$. This implies that for the disease to persist for each larval mosquito produced, $1 /\left(1-q_{v}\right)$ should be infected. This explicit formulation of the threshold conditions in terms of parameters governing the infection transmission process and vector life history is of great epidemiological significance as it allows disease control efforts to be targeted at specific disease and vector stages. We have also shown that the disease-free equilibrium is locally asymptotically stable for $R_{0}^{c}<1$ and globally asymptotically stable if $R_{0}^{c} \leqslant 1$. The latter result is of outmost importance, because it shows that if at any time, through appropriate interventions (e.g., destruction of breeding sites, use of insecticides and repellents on the host, or even vaccination), we are able to lower $R_{0}^{c}$ below 1 , then the disease will disappear. When the vertical transmission route is possible, this requires also lowering the contribution from this transmission mode. That is, lowering $Q_{0}^{v}$ which implies keeping $q_{v}$ very close to zero. Therefore, the design of control programs should take into account the implications of this mode of transmission in the case of vector-borne diseases that include vertical transmission in the vector population.

The goal of this study was to use mathematical models for discussing important properties of vector-borne disease systems and their implications in attempts for control strategies. Though we have used simple but realistic models, we believe that they remain valuable tools useful in the context of research and for providing qualitative understanding of complex processes underlying vector-borne disease transmission systems in particular in the context of transovarial transmission in the vector population. In conclusion, our results show that (1) including larval stages in the vector population when modelling vector-borne disease systems has benefits, as it allows determining conditions for colonization in terms of vector life cycle features; (2) the failure of $R_{0}$ derived from the next-generation method compounds itself in the presence of vertical transmission; (3) host type reproductive number gives the correct $R_{0}$ useful for guiding disease control strategies; (4) while vertical transmission efficiency is likely to increase the size of an epidemic, it decreases its duration; and (5) in the presence of vertical infection the critical ratio of female mosquitoes to hosts reduces linearly when increasing vertical transmission efficiency.

\section{Data Availability}

The relevant data used to support the findings of this study are included within the article.

\section{Conflicts of Interest}

The author declares that he has no conflicts of interest.

\section{Supplementary Materials}

Supplementary material contains details on the analytical derivation of the basic reproductive number, type reproductive numbers, and model equilibria. Table 1: parameter values used in the simulations. (Supplementary Materials)

\section{References}

[1] D. J. Gubler, "Vector-borne diseases," Revue Scientifique et Technique de l'OIE, vol. 28, no. 2, pp. 583-588, 2009.

[2] World Health Organization, WHO-Vector-Borne Diseases, 2016.

[3] A. Huppert and G. Katriel, "Mathematical modelling and prediction in infectious disease epidemiology," Clinical Microbiology and Infection, vol. 19, no. 11, pp. 999-1005, 2013.

[4] F. Brauer, "Mathematical epidemiology: Past, present, and future," Infectious Disease Modelling, vol. 2, no. 2, pp. 113-127, 2017.

[5] C. E. Walters, M. M. Meslé, and I. M. Hall, "Modelling the global spread of diseases: A review of current practice and capability," Epidemics, 2018.

[6] R. M. Murithi, P. Munyua, P. M. Ithondeka et al., "Rift Valley fever in Kenya: History of epizootics and identification of vulnerable districts," Epidemiology and Infection, vol. 139, no. 3, pp. 372-380, 2011.

[7] C. Sindato, E. D. Karimuribo, D. U. Pfeiffer et al., "Spatial and temporal pattern of rift valley fever outbreaks in Tanzania; 1930 to 2007," PLoS ONE, vol. 9, no. 2, 2014.

[8] K. J. Linthicum, F. G. Davies, A. Kairo, and C. L. Bailey, "Rift Valley fever virus (family Bunyaviridae, genus Phlebovirus). Isolations from diptera collected during an inter-epizootic period in Kenya, Epidemiology \& Infection, vol. 95, no. 1, pp. 197-209, 1985.

[9] W. Horsfall, H. Fowler, M. Lj, and L. Jr, Bionomics and Embryology of the Inland Flood Water Mosquito Aedes Vexans, University of Illinois Press, 1974.

[10] F. F. Ludueña Almeida and D. E. Gorla, "The biology of Aedes (Ochlerotatus) albifasciatus Macquart, 1838 (Diptera: 
Culicidae) in central Argentina," Memórias do Instituto Oswaldo Cruz, vol. 90, no. 4, pp. 463-468, 1995.

[11] N. Becker, D. Petric, M. Zgomba et al., Mosquitoes and Their Control, Springer-Verlag Berlin Heidelberg 2003, 2010.

[12] K. J. Linthicum, T. M. Logan, C. L. Bailey, D. J. Dohm, and J. R. Moulton, "Transstadial and horizontal transmission of Rift Valley fever virus in Hyalomma truncatum," The American Journal of Tropical Medicine and Hygiene, vol. 41, no. 4, pp. 491496, 1989.

[13] X. Guo, T. Zhao, Y. Dong, and B. Lu, "Survival and replication of dengue-2 virus in diapausing eggs of Aedes albopictus (diptera: Culicidae)," Journal of Medical Entomology, vol. 44, no. 3, pp. 492-497, 2007.

[14] B. Angel and V. Joshi, "Distribution and seasonality of vertically transmitted dengue viruses in Aedes mosquitoes in arid and semi-arid areas of Rajasthan, India," Journal of Vector Borne Diseases, vol. 45, no. 1, pp. 56-59, 2008.

[15] M. Pepin, M. Bouloy, B. H. Bird, A. Kemp, and J. Paweska, "Rift Valley fever virus (Bunyaviridae: Phlebovirus): An update on pathogenesis, molecular epidemiology, vectors, diagnostics and prevention," Veterinary Research, vol. 41, no. 6, 2010.

[16] Y. Ba, D. Diallo, C. M. F. Kebe, I. Dia, and M. Diallo, "Aspects of bioecology of two rift valley fever virus vectors in Senegal (West Africa): Aedes vexans and Culex poicilipes (Diptera: Culicidae)," Journal of Medical Entomology, vol. 42, no. 5, pp. 739-750, 2005.

[17] A. D. Labeaud, E. M. Muchiri, M. Ndzovu et al., "Interepidemic Rift Valley fever virus seropositivity, northeastern Kenya," Emerging Infectious Diseases, vol. 14, no. 8, pp. 1240-1246, 2008.

[18] R. D. Sumaye, E. Geubbels, E. Mbeyela, and D. Berkvens, "Inter-epidemic transmission of rift valley fever in livestock in the Kilombero river valley, Tanzania: a cross-sectional survey," PLOS Neglected Tropical Diseases, vol. 7, no. 8, Article ID e2356, 2013.

[19] N. O. Owange, W. O. Ogara, H. Affognon et al., "Occurrence of rift valley fever in cattle in Ijara district, Kenya," Preventive Veterinary Medicine, vol. 117, no. 1, pp. 121-128, 2014.

[20] J. K. Lichoti, A. Kihara, A. A. Oriko et al., "Detection of rift valley fever virus interepidemic activity in some hotspot areas of kenya by sentinel animal surveillance, 2009-2012," Veterinary Medicine international, vol. 2014, Article ID 379010, 9 pages, 2014.

[21] J. Li, D. Blakeley, and R. J. Smith, “The failure of $R_{0}$ " Computational and Mathematical Methods in Medicine, vol. 2011, Article ID 527610, 17 pages, 2011.

[22] H. D. Gaff, D. M. Hartley, and N. P. Leahy, "An epidemiological model of rift valley fever," Electronic Journal of Differential Equations, vol. 115, pp. 1-12, 2007.

[23] B. Adams and M. Boots, "How important is vertical transmission in mosquitoes for the persistence of dengue? Insights from a mathematical model," Epidemics, vol. 2, no. 1, pp. 1-10, 2010.

[24] N. Chitnis, J. M. Hyman, and C. A. Manore, "Modelling vertical transmission in vector-borne diseases with applications to Rift Valley fever," Journal of Biological Dynamics, vol. 7, no. 1, pp. 1140, 2013.

[25] S. A. Pedro, S. Abelman, F. T. Ndjomatchoua, R. Sang, and H. E. Z. Tonnang, "Stability, bifurcation and chaos analysis of vectorborne disease model with application to rift valley fever," PLOS ONE, vol. 9, no. 10, Article ID e108172, 2014.

[26] F. Chamchod, R. S. Cantrell, C. Cosner, A. N. Hassan, J. C. Beier, and S. Ruan, "A modeling approach to investigate epizootic outbreaks and enzootic maintenance of Rift Valley fever virus," Bulletin of Mathematical Biology, vol. 76, no. 8, pp. 2052-2072, 2014.

[27] S. A. Pedro, H. E. Tonnang, and S. Abelman, "Uncertainty and sensitivity analysis of a Rift Valley fever model," Applied Mathematics and Computation, vol. 279, pp. 170-186, 2016.

[28] S. A. Pedro, S. Abelman, and H. E. Z. Tonnang, "Predicting Rift Valley Fever Inter-epidemic Activities and Outbreak Patterns: Insights from a Stochastic Host-Vector Model," PLOS Neglected Tropical Diseases, vol. 10, no. 12, 2016.

[29] R. Ross, Murray London, 2nd edition, 1911.

[30] G. Macdonald, “The analysis of equilibrium in malaria," Tropical Diseases Bulletin, vol. 49, no. 9, pp. 813-829, 1952.

[31] R. M. Anderson, Population Dynamics of Infectious Diseases: Theory and Applications, vol. 368, 1982.

[32] N. A. Maidana and H. M. Yang, "Describing the geographic spread of dengue disease by traveling waves," Mathematical Biosciences, vol. 215, no. 1, pp. 64-77, 2008.

[33] H. R. Thieme, Mathematics in Population Biology, vol. 47, 2003.

[34] J. LaSalle, "The stability of dynamical systems," in Proceedings of the CBMS-NSF Regional Conference Series in Applied Mathematics, SIAM, Thailand, Philadelphia, USA, 1976.

[35] O. Diekmann and J. A. P. Heesterbeek, Mathematical Epidemiology of Infectious Diseases: Model Building, Analysis and Interpretation, John Wiley \& Sons, 2000.

[36] P. van den Driessche and J. Watmough, "Reproduction numbers and sub-threshold endemic equilibria for compartmental models of disease transmission," Mathematical Biosciences, vol. 180, pp. 29-48, 2002.

[37] M. J. Keeling and P. Rohani, Modeling Infectious Diseases in Humans and Animals, vol. 47, 2007.

[38] M. G. Roberts and J. A. P. Heesterbeek, "A new method for estimating the effort required to control an infectious disease," Proceedings of the Royal Society B Biological Science, vol. 270, no. 1522, pp. 1359-1364, 2003.

[39] J. A. Heesterbeek and M. G. Roberts, "The type-reproduction number $\mathrm{t}$ in models for infectious disease control," Mathematical Biosciences, vol. 206, no. 1, pp. 3-10, 2007.

[40] J. C. Kamgang and G. Sallet, "Global asymptotic stability for the disease free equilibrium for epidemiological models," Comptes Rendus Mathematique, vol. 341, no. 7, pp. 433-438, 2005.

[41] C. Castillo-Chavez and B. Song, "Dynamical models of tuberculosis and their applications," Mathematical Biosciences and Engineering, vol. 1, no. 2, pp. 361-404, 2004.

[42] J. M. Heffernan, R. J. Smith, and L. M. Wahl, "Perspectives on the basic reproductive ratio," Journal of the Royal Society Interface, vol. 2, no. 4, pp. 281-293, 2005.

[43] A. L. Lloyd, J. Zhang, and A. M. Root, "Stochasticity and heterogeneity in host-vector models," Journal of the Royal Society Interface, vol. 4, no. 16, pp. 851-863, 2007. 


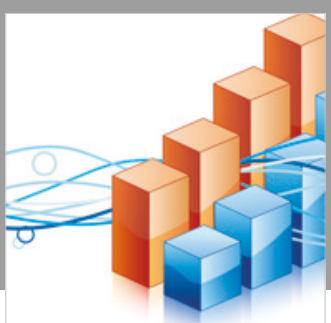

Advances in

Operations Research

\section{-n-m}
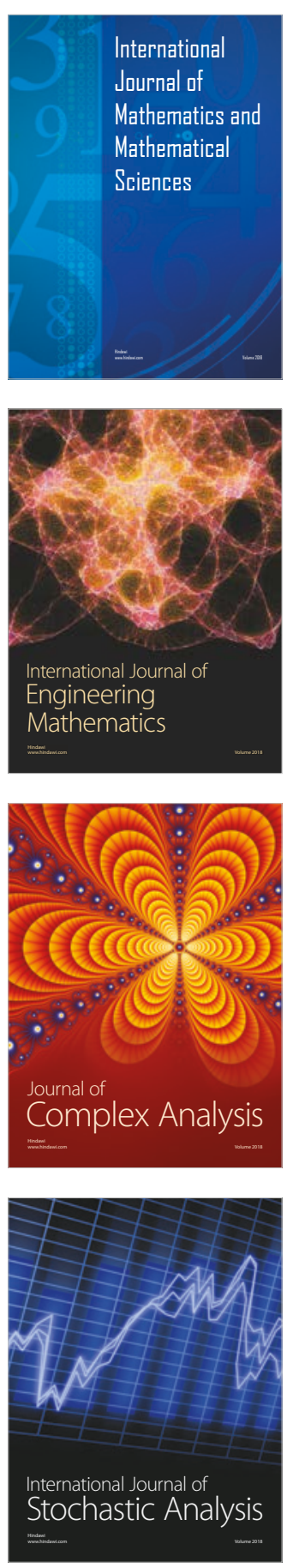
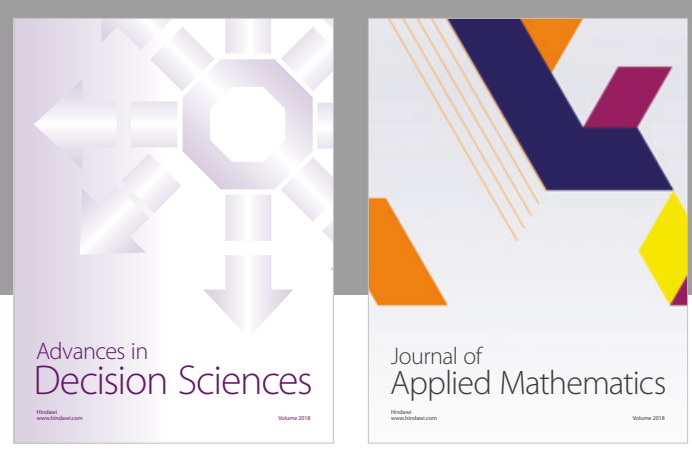

Journal of

Applied Mathematics
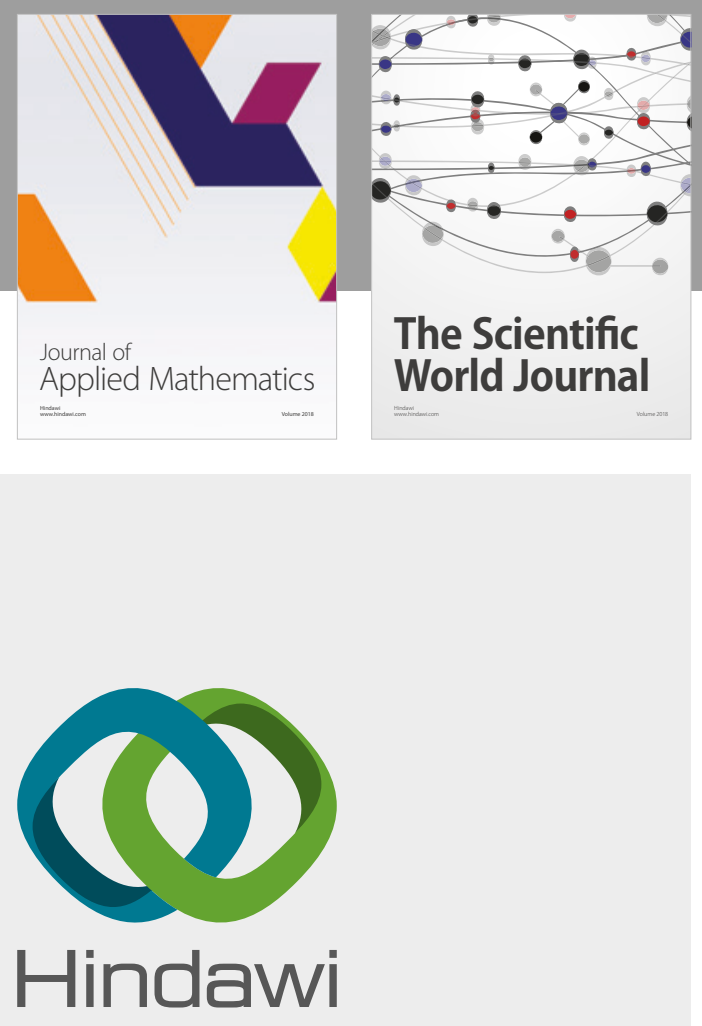

Submit your manuscripts at

www.hindawi.com

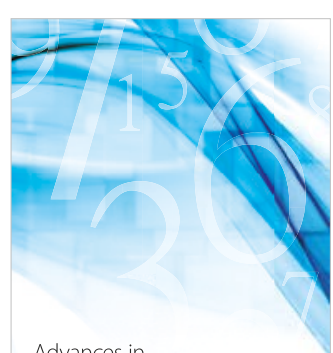

Advances in
Numerical Analysis
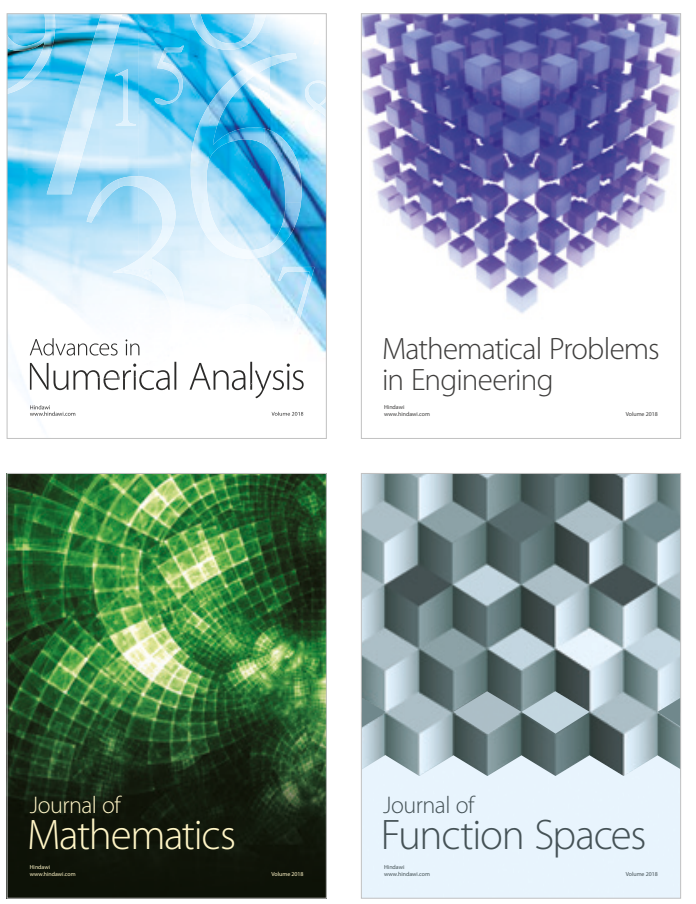

Mathematical Problems in Engineering

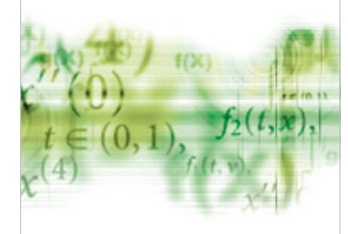

International Journal of

Differential Equations

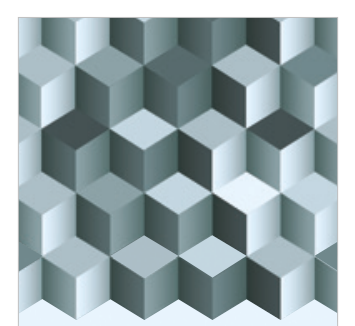

Journal of

Function Spaces

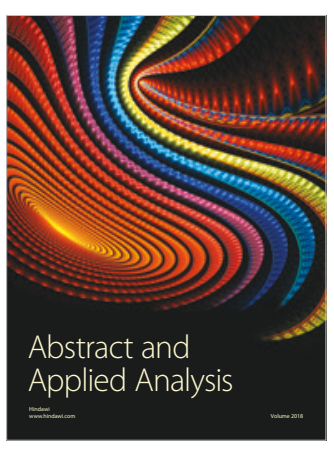

The Scientific

World Journal

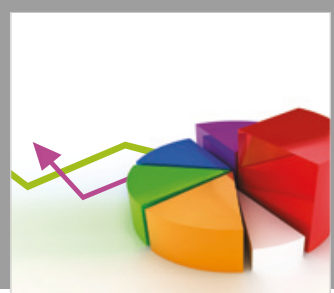

Journal of

Probability and Statistics
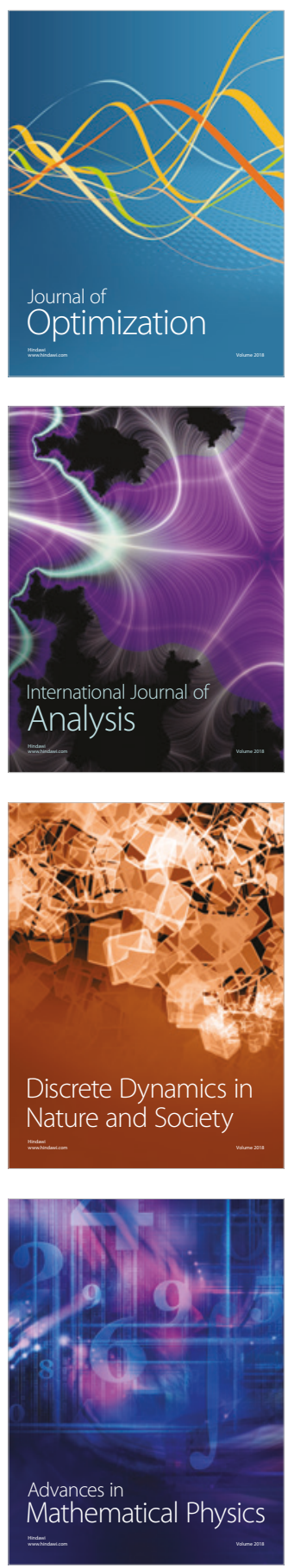